\title{
Phase Separation in Charge-Stabilized Colloidal Suspensions: Influence of Nonlinear Screening
}

\author{
A. R. Dentor* \\ Department of Physics, North Dakota State University, Fargo, ND, 58105-5566
}

(Dated: April 24, 2018)

\begin{abstract}
The phase behavior of charge-stabilized colloidal suspensions is modeled by a combination of response theory for electrostatic interparticle interactions and variational theory for free energies. Integrating out degrees of freedom of the microions (counterions, salt ions), the macroion-microion mixture is mapped onto a one-component system governed by effective macroion interactions. Linear response of microions to the electrostatic potential of the macroions results in a screened-Coulomb (Yukawa) effective pair potential and a one-body volume energy, while nonlinear response modifies the effective interactions [A. R. Denton, Phys. Rev. E 70, 031404 (2004)]. The volume energy and effective pair potential are taken as input to a variational free energy, based on thermodynamic perturbation theory. For both linear and first-order nonlinear effective interactions, a coexistence analysis applied to aqueous suspensions of highly charged macroions and monovalent microions yields bulk separation of macroion-rich and macroion-poor phases below a critical salt concentration, in qualitative agreement with predictions of related linearized theories [R. van Roij, M. Dijkstra, and J.-P. Hansen, Phys. Rev. E 59, 2010 (1999); P. B. Warren, J. Chem. Phys. 112, 4683 (2000)]. It is concluded that nonlinear screening can modify phase behavior but does not necessarily suppress bulk phase separation of deionized suspensions.
\end{abstract}

\section{INTRODUCTION}

Mounting evidence from a variety of experiments suggests that colloidal suspensions [1, 2, 3] of highly charged macroions and monovalent microions (counterions and coions) can separate into macroion-rich and -poor bulk phases at low salt concentrations. Reported observations - in aqueous suspensions at submillimolar ionic strengths - describe liquid-vapor coexistence [4], stable voids [5, 6, 7, 8], contracted crystal lattices [8, 9, 10], and metastable crystallites [11]. Such phenomena suggest an unusual form of interparticle cohesion, inconsistent with the long-ranged repulsive electrostatic pair interactions that prevail at low ionic strengths 12, and in apparent conflict with the classic theory of Derjaguin, Landau, Verwey, and Overbeek (DLVO) 13, 14], which so successfully describes phase stability with respect to coagulation at higher salt concentrations. Observations of bulk phase separation in deionized suspensions are therefore often considered anomalous.

Reports of anomalous phase behavior in charged colloids have been variously disputed [15], attributed to impurities 16, 17], or interpreted as genuine manifestations of like-charge interparticle attraction 4, 5, 6, 7, 8, 9, 10, 11], whether pairwise or many-body in origin 18, 19, 20]. Although some particle-tracking experiments [11, 21, 22, 23] appear to exhibit attractive forces between isolated pairs of tightly confined macroions, recent studies, based on refined optical imaging methods, have found no attraction 24]. Furthermore, mathematical proofs that Poisson-Boltzmann theory predicts purely repulsive pair interactions 25, 26, 27] relegate any possible pair attraction to the influence of counterion correlations, neglected by the mean-field theory. It is now widely accepted that correlations among multivalent counterions can induce attraction between like-charged surfaces 28, 29, 30, 31, 32], as well as condensation of DNA and other polyelectrolytes [32, 33, 34, 35, 36, 37, 38, 39, 40, 41]. The key issue motivating the present study is whether relatively weakly correlated monovalent counterions can similarly destabilize deionized colloidal suspensions.

Further evidence for effective attractive interactions in charged colloids comes from computer simulations. Monte Carlo simulations [42, 43, 44, 45] of the primitive model of asymmetric electrolytes - macroions and microions, in a dielectic continuum, directly interacting via repulsive Coulomb pair potentials - ex-

*Electronic address: alan.denton@ndsu.edu 
hibit macroion attraction and instabilities toward macroion aggregation at high electrostatic couplings. Short-ranged attractions have been linked to spatial correlations among counterions localized near different macroions [42, 43], or to Coulomb depletion [4], while long-ranged attractions have been attributed to overcharging of macroions [45]. System parameters thus far explored correspond to relatively strongly correlated (multivalent) counterions and relatively small macroion-to-counterion size and charge asymmetries. Computational advances, however, are rapidly closing the gap that currently prevents direct comparison of simulations and experiments.

Many theoretical studies of interparticle interactions and phase behavior in charged colloids have been motivated by the puzzling results of experiments and simulations. Among various analytical and computational approaches, recently reviewed [16, 46, 47, 48], are integral-equation, Poisson-Boltzmann, density-functional, Debye-Hückel, and response theories. In seminal work, van Roij et al. [49, 50, 51, 52], described the phase behavior of charged colloids within an effective one-component model governed by density-dependent effective interactions. Combining a linearized density-functional theory [53] for the effective pair and one-body (volume energy) potentials with a variational theory for the free energy, these authors predicted counterion-driven bulk phase separation in deionized suspensions of highly charged macroions below a critical salt concentration. Subsequently, Warren [54] applied an extended Debye-Hückel (linearized Poisson-Boltzmann) theory and predicted similarly unusual phase separation at low salt concentrations. Statistical mechanical [55, 56] and linear-response [57, 58, 59] methods, based on closely related linearization approximations, yield similar effective electrostatic interactions.

Several recent studies, based on Poisson-Boltzmann cell models 60, 61, 62 and extensions of Debye-Hückel theory [63], have suggested that predicted instabilities of charged colloids towards phase separation may be mere artifacts of linearization. The main purpose of the present study is to directly test this suggestion by explicitly calculating the effect of nonlinear screening on the phase behavior of charged colloids. Working within the framework of the effective one-component model and response theory [57, 58, 59], we input nonlinear corrections to the effective pair potential and volume energy into an accurate variational free energy and analyze thermodynamic phase behavior. The central conclusion of the paper is that nonlinear effects can modify phase behavior of deionized suspensions, but do not necessarily suppress counterion-driven phase separation.

Outlining the remainder of the paper, Sec. Ifirst defines the model colloidal suspension. Section III next reviews the response theory for effective interactions and describes a variational perturbation theory for the free energy. Section IV presents and discusses numerical results - most importantly, equilibrium phase diagrams obtained from a coexistence analysis. Finally, Sec. $\nabla$ summarizes and concludes.

\section{MODEL SYSTEM}

The system of interest comprises $N_{m}$ negatively charged colloidal macroions, $N_{c}$ positively charged counterions, and $N_{s}$ pairs of oppositely charged salt ions all dispersed in a solvent. The macroions are modeled as charged hard spheres of radius $a$ (diameter $\sigma$ ) and effective valence $Z$, as depicted in Fig. 1. The macroion surface charge $-Z e$ is best interpreted as an effective (renormalized) charge, equal to the bare charge less the combined charge of any strongly associated counterions. The effective charge is assumed to be uniformly distributed over the surface and fixed, independent of thermodynamic state. The counterions and salt ions are modeled as point charges of valence $z$, whose number $N_{c}$ is determined by the condition of overall charge neutrality: $Z N_{m}=z N_{c}$. Numerical results are presented below (Sec. IV] for the case of monovalent $(z=1)$ microions. The microions number $N_{+}=N_{c}+N_{s}$ positive and $N_{-}=N_{s}$ negative, totaling $N_{\mu}=N_{c}+2 N_{s}$.

Working within the primitive model of charged colloids, we approximate the solvent as a dielectric continuum, characterized entirely by a dielectric constant $\epsilon$. We further assume a rigid-ion model, ignoring van der Waals [12] and polarization [64, 65, 66] interactions, which are dominated by longer-ranged direct electrostatic interactions at low ionic strengths. The system is imagined to be in thermal equilibrium with a heat bath at constant temperature and in chemical (Donnan) equilibrium with a salt reservoir (e.g., via a semi-permeable membrane or ion-exchange resin), which fixes the salt chemical potential. Having specified the model system, we turn next to methods for describing electrostatic interactions and thermodynamic phase behavior. 


\section{METHODS}

\section{A. Effective Electrostatic Interactions}

\section{One-Component Mapping}

Response theory of effective interactions is fundamentally based on mapping a multi-component mixture onto a one-component system governed by an effective Hamiltonian [67]. When applied to charged colloids, polyelectrolytes, and other ionic systems, the mapping involves integrating out from the partition function the degrees of freedom of the microions [68]. The resulting effective interactions between macroions depend on the perturbation of the microion distribution by the "external" potential of the macroions. The response of the microions to the macroions is linear [57, 58] for suspensions of weakly charged macroions, but becomes increasingly nonlinear [59] as the macroion valence increases and as the salt concentration decreases. Here we briefly review the theory, referring the reader to refs. [57, 58, 59] for further details.

In the simplest case of a salt-free suspension, the Hamiltonian may be expressed as

$$
H=H_{m}(\{\mathbf{R}\})+H_{c}(\{\mathbf{r}\})+H_{m c}(\{\mathbf{R}\},\{\mathbf{r}\}),
$$

where $\{\mathbf{R}\}$ and $\{\mathbf{r}\}$ denote coordinates of macroions and microions, respectively,

$$
H_{m}=H_{\mathrm{HS}}(\{\mathbf{R}\})+\frac{1}{2} \sum_{i \neq j=1}^{N_{m}} v_{m m}\left(\left|\mathbf{R}_{i}-\mathbf{R}_{j}\right|\right)
$$

is the Hamiltonian of the macroions alone, $H_{\mathrm{HS}}$ is the Hamiltonian of a hard-sphere (HS) system, $v_{m m}(r)=$ $Z^{2} e^{2} / \epsilon r, r>\sigma$, is the bare Coulomb pair potential between macroions,

$$
H_{c}=K_{c}+\frac{1}{2} \sum_{i \neq j=1}^{N_{c}} v_{c c}\left(\left|\mathbf{r}_{i}-\mathbf{r}_{j}\right|\right)
$$

is the counterion Hamiltonian, $K_{c}$ is the counterion kinetic energy, $v_{c c}(r)=z^{2} e^{2} / \epsilon r$ is the pair potential between counterions,

$$
H_{m c}=\sum_{i=1}^{N_{m}} \sum_{j=1}^{N_{c}} v_{m c}\left(\left|\mathbf{R}_{i}-\mathbf{r}_{j}\right|\right)
$$

is the total macroion-counterion interaction energy, and $v_{m c}(r)=Z z e^{2} / \epsilon r, r>a$, is the macroion-counterion pair potential.

The mapping from the macroion-counterion mixture to an effective one-component system of pseudomacroions begins with the canonical partition function

$$
\mathcal{Z}=\left\langle\langle\exp (-\beta H)\rangle_{c}\right\rangle_{m},
$$

where $\beta \equiv 1 /\left(k_{B} T\right)$ at temperature $T$ and angular brackets denote classical traces over counterion $(c)$ and macroion $(m)$ coordinates. The mapping proceeds by formally tracing over the counterion coordinates:

$$
\mathcal{Z}=\left\langle\exp \left(-\beta H_{\mathrm{eff}}\right)\right\rangle_{m}
$$

where $H_{\text {eff }}=H_{m}+F_{c}$ is the effective one-component Hamiltonian and

$$
F_{c}=-k_{B} T \ln \left\langle\exp \left[-\beta\left(H_{c}+H_{m c}\right)\right]\right\rangle_{c}
$$

is the free energy of a nonuniform gas of counterions in the presence of the fixed macroions. Within perturbation theory [68, [69], the counterion free energy can be expressed as

$$
F_{c}=F_{0}+\int_{0}^{1} \mathrm{~d} \lambda \frac{\partial F_{c}(\lambda)}{\partial \lambda}=F_{0}+\int_{0}^{1} \mathrm{~d} \lambda\left\langle H_{m c}\right\rangle_{\lambda}
$$


where $F_{c}(\lambda) \equiv-k_{B} T \ln \left\langle\exp \left[-\beta\left(H_{c}+\lambda H_{m c}\right)\right]\right\rangle_{c}, F_{0} \equiv F_{c}(0)=-k_{B} T \ln \left\langle\exp \left(-\beta H_{c}\right)\right\rangle$ is the unperturbed counterion free energy in the case of uncharged (yet volume-excluding) macroions, \langle\rangle$_{\lambda}$ denotes a counterion trace with the macroions charged to a fraction $\lambda$ of their full charge, and the $\lambda$-integral charges up the macroions. After formally adding and subtracting the energy of a uniform compensating negative background $E_{b}$, Eq. (8) becomes

$$
F_{c}=F_{\mathrm{OCP}}+\int_{0}^{1} \mathrm{~d} \lambda\left\langle H_{m c}\right\rangle_{\lambda}-E_{b},
$$

where $F_{\mathrm{OCP}}=F_{0}+E_{b}$ is the free energy of a classical one-component plasma (OCP) in the presence of neutral hard spheres. The background and counterions alike are excluded from the hard cores of the macroions and therefore occupy a free volume $V^{\prime}=V(1-\eta)$, where $\eta=(\pi / 6)\left(N_{m} / V\right) \sigma^{3}$ is the macroion volume fraction.

\section{Response Theory}

To make practical use of the one-component mapping, the counterion free energy must be approximated, for which purpose response theory provides a powerful framework. Because it proves more convenient to manipulate Fourier components of densities and pair potentials, we first note that the macroion Hamiltonian [Eq. (2)] and macroion-counterion interaction [Eq. (4)] can be equivalently expressed as

$$
H_{m}=H_{\mathrm{HS}}+\frac{1}{2 V^{\prime}} \sum_{\mathbf{k}} \hat{v}_{m m}(k)\left[\hat{\rho}_{m}(\mathbf{k}) \hat{\rho}_{m}(-\mathbf{k})-N_{m}\right]
$$

and

$$
H_{m c}=\frac{1}{V^{\prime}} \sum_{\mathbf{k}} \hat{v}_{m c}(k) \hat{\rho}_{m}(\mathbf{k}) \hat{\rho}_{c}(-\mathbf{k}),
$$

where the Fourier transform and its inverse are defined as

$$
\begin{gathered}
\hat{\rho}_{m}(\mathbf{k})=\int \mathrm{d} \mathbf{r} \rho_{m}(\mathbf{r}) e^{-i \mathbf{k} \cdot \mathbf{r}} \\
\rho_{m}(\mathbf{r})=\frac{1}{V^{\prime}} \sum_{\mathbf{k}} \hat{\rho}_{m}(\mathbf{k}) e^{i \mathbf{k} \cdot \mathbf{r}}
\end{gathered}
$$

Equation (11) makes evident that $H_{m c}$ depends, through $\hat{\rho}_{c}(\mathbf{k})$, on the response of the counterion density to the macroion charge density. The counterion response can be approximated by expanding the ensembleaveraged induced counterion density in a functional Taylor series in powers of the dimensionless macroion potential 69, 70, 71, 72], $u(\mathbf{r})=-\beta \int \mathrm{d} \mathbf{r}^{\prime} v_{m c}\left(\left|\mathbf{r}-\mathbf{r}^{\prime}\right|\right) \rho_{m}\left(\mathbf{r}^{\prime}\right)$. Expanding about zero macroion charge $(u=0)$, the counterion density can be expressed, in Fourier space, as [59]

$$
\begin{aligned}
\left\langle\hat{\rho}_{c}(\mathbf{k})\right\rangle & =\chi(k) \hat{v}_{m c}(k) \hat{\rho}_{m}(\mathbf{k})+\frac{1}{V^{\prime}} \sum_{\mathbf{k}^{\prime}} \chi^{\prime}\left(\mathbf{k}^{\prime}, \mathbf{k}-\mathbf{k}^{\prime}\right) \hat{v}_{m c}\left(k^{\prime}\right) \hat{v}_{m c}\left(\left|\mathbf{k}-\mathbf{k}^{\prime}\right|\right) \\
& \times \hat{\rho}_{m}\left(\mathbf{k}^{\prime}\right) \hat{\rho}_{m}\left(\mathbf{k}-\mathbf{k}^{\prime}\right)+\cdots, \quad k \neq 0,
\end{aligned}
$$

where $\chi$ and $\chi^{\prime}$ are, respectively, the linear and first nonlinear response functions of the uniform OCP. The response functions are directly related to the structure of the OCP according to $\chi(k)=-\beta n_{c} S(k)$ and $\chi^{\prime}\left(\mathbf{k}^{\prime}, \mathbf{k}-\mathbf{k}^{\prime}\right)=\left(\beta^{2} n_{c} / 2\right) S^{(3)}\left(\mathbf{k}^{\prime}, \mathbf{k}-\mathbf{k}^{\prime}\right)$, where

$$
S^{(n)}\left(\mathbf{k}_{1}, \cdots, \mathbf{k}_{n-1}\right)=\frac{1}{N_{c}}\left\langle\hat{\rho}_{c}\left(\mathbf{k}_{1}\right) \cdots \hat{\rho}_{c}\left(\mathbf{k}_{n-1}\right) \hat{\rho}_{c}\left(-\mathbf{k}_{1} \ldots-\mathbf{k}_{n-1}\right)\right\rangle
$$

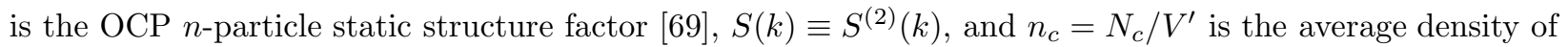
counterions in the free volume. The first term on the right side of Eq. (14), which is linear in $\hat{v}_{m c}(k)$ and $\hat{\rho}_{m}(\mathbf{k})$, represents the linear response approximation, while the higher-order terms are nonlinear corrections. 
Combining Eqs. (9), (11), and (14), specifying the background energy as $E_{b}=\lim _{k \rightarrow 0}\left\{-N_{c} n_{c} \hat{v}_{c c}(k) / 2\right\}$, isolating the $k=0$ terms, and integrating over $\lambda$, produces the counterion free energy to third order in the macroion density:

$$
\begin{gathered}
F_{c}=F_{\mathrm{OCP}}+n_{c} \lim _{k \rightarrow 0}\left[N_{m} \hat{v}_{m c}(k)+\frac{N_{c}}{2} \hat{v}_{c c}(k)\right]+\frac{1}{2 V^{\prime}} \sum_{\mathbf{k} \neq 0} \chi(k)\left[\hat{v}_{m c}(k)\right]^{2} \hat{\rho}_{m}(\mathbf{k}) \hat{\rho}_{m}(-\mathbf{k}) \\
+\frac{1}{3 V^{\prime 2}} \sum_{\mathbf{k} \neq 0} \sum_{\mathbf{k}^{\prime}} \chi^{\prime}\left(\mathbf{k}^{\prime},-\mathbf{k}-\mathbf{k}^{\prime}\right) \hat{v}_{m c}(k) \hat{v}_{m c}\left(k^{\prime}\right) \hat{v}_{m c}\left(\left|\mathbf{k}+\mathbf{k}^{\prime}\right|\right) \hat{\rho}_{m}(\mathbf{k}) \hat{\rho}_{m}\left(\mathbf{k}^{\prime}\right) \hat{\rho}_{m}\left(-\mathbf{k}-\mathbf{k}^{\prime}\right) .
\end{gathered}
$$

The terms in $F_{c}$ that are quadratic and cubic in $\hat{\rho}_{m}(\mathbf{k})$ generate effective pair and triplet interactions, respectively, in the effective Hamiltonian. To demonstrate this, we first identify

$$
\hat{v}_{\text {ind }}^{(2)}(k)=\chi(k)\left[\hat{v}_{m c}(k)\right]^{2}
$$

as an effective pair interaction, induced by linear response of counterions [57, [58, 68], and

$$
\hat{v}_{\mathrm{eff}}^{(3)}\left(\mathbf{k}, \mathbf{k}^{\prime}\right)=2 \chi^{\prime}\left(\mathbf{k}^{\prime},-\mathbf{k}-\mathbf{k}^{\prime}\right) \hat{v}_{m c}(k) \hat{v}_{m c}\left(k^{\prime}\right) \hat{v}_{m c}\left(\left|\mathbf{k}+\mathbf{k}^{\prime}\right|\right)
$$

as an effective three-body interaction, induced by nonlinear counterion response. Combining Eqs. (10) and (16), the effective Hamiltonian now can be recast in the form

$$
H_{\mathrm{eff}}=H_{\mathrm{HS}}+\frac{1}{2} \sum_{i \neq j=1}^{N_{m}} v_{\mathrm{eff}}^{(2)}\left(\left|\mathbf{R}_{i}-\mathbf{R}_{j}\right|\right)+\frac{1}{3 !} \sum_{i \neq j \neq k=1}^{N_{m}} v_{\mathrm{eff}}^{(3)}\left(\mathbf{R}_{i}-\mathbf{R}_{j}, \mathbf{R}_{i}-\mathbf{R}_{k}\right)+E,
$$

where $v_{\text {eff }}^{(2)}(r)=v_{m m}(r)+v_{\text {ind }}^{(2)}(r)$ and $v_{\text {eff }}^{(3)}\left(\mathbf{r}, \mathbf{r}^{\prime}\right)$ are the effective macroion pair and triplet potentials, respectively, and $E$ is a one-body volume energy, composed of all terms in $H_{\text {eff }}$ independent of macroion coordinates. The volume energy accounts for the counterion entropy and macroion-counterion interaction energy and contributes density-dependent terms to the total free energy that can influence thermodynamic properties, as discussed below (Sec. IV).

Explicit expressions for the effective interactions are obtained by invoking the identities

$$
\sum_{i \neq j=1}^{N_{m}} v_{\text {ind }}^{(2)}\left(\left|\mathbf{R}_{i}-\mathbf{R}_{j}\right|\right)=\frac{1}{V^{\prime}} \sum_{\mathbf{k} \neq 0} \hat{v}_{\text {ind }}^{(2)}(k) \hat{\rho}_{m}(\mathbf{k}) \hat{\rho}_{m}(-\mathbf{k})+\frac{N_{m}^{2}}{V^{\prime}} \lim _{k \rightarrow 0} \hat{v}_{\text {ind }}^{(2)}(k)-N_{m} v_{\text {ind }}^{(2)}(0)
$$

and

$$
\begin{aligned}
\sum_{i \neq j \neq k=1}^{N_{m}} v_{\mathrm{eff}}^{(3)}\left(\mathbf{R}_{i}-\mathbf{R}_{j}, \mathbf{R}_{i}-\mathbf{R}_{k}\right) & =\frac{1}{V^{\prime 2}} \sum_{\mathbf{k}} \sum_{\mathbf{k}^{\prime}} \hat{v}_{\mathrm{eff}}^{(3)}\left(\mathbf{k}, \mathbf{k}^{\prime}\right)\left[\hat{\rho}_{m}(\mathbf{k}) \hat{\rho}_{m}\left(\mathbf{k}^{\prime}\right) \hat{\rho}_{m}\left(-\mathbf{k}-\mathbf{k}^{\prime}\right)\right. \\
& \left.-3 \hat{\rho}_{m}(\mathbf{k}) \hat{\rho}_{m}(-\mathbf{k})+2 N_{m}\right] .
\end{aligned}
$$

The volume energy, $E=E_{0}+\Delta E$, is the sum of the linear response approximation [57, 58.

$$
E_{0}=F_{\mathrm{OCP}}+\frac{N_{m}}{2} v_{\text {ind }}^{(2)}(0)+N_{m} n_{c} \lim _{k \rightarrow 0}\left[\hat{v}_{m c}(k)-\frac{z}{2 Z} \hat{v}_{\text {ind }}^{(2)}(k)+\frac{Z}{2 z} \hat{v}_{c c}(k)\right]
$$

and the first nonlinear correction $[59$

$$
\Delta E=\frac{N_{m}}{6 V^{\prime 2}}\left[\sum_{\mathbf{k}, \mathbf{k}^{\prime}} \hat{v}_{\mathrm{eff}}^{(3)}\left(\mathbf{k}, \mathbf{k}^{\prime}\right)-N_{m} \sum_{\mathbf{k}} \hat{v}_{\mathrm{eff}}^{(3)}(\mathbf{k}, 0)\right] .
$$

Similarly, the effective pair interaction, $v_{\mathrm{eff}}^{(2)}(r)=v_{0}^{(2)}(r)+\Delta v_{\mathrm{eff}}^{(2)}(r)$ is the sum of the linear response approximation [57, 58], $v_{0}^{(2)}(r)=v_{m m}(r)+v_{\text {ind }}^{(2)}(r)$, and the first nonlinear correction, $\Delta v_{\text {eff }}^{(2)}(r)$, whose Fourier transform is

$$
\Delta \hat{v}_{\mathrm{eff}}^{(2)}(k)=\frac{1}{V^{\prime}} \sum_{\mathbf{k}^{\prime}} \hat{v}_{\mathrm{eff}}^{(3)}\left(\mathbf{k}, \mathbf{k}^{\prime}\right)-\frac{N_{m}}{3 V^{\prime}} \hat{v}_{\mathrm{eff}}^{(3)}(\mathbf{k}, 0)
$$


It is important to note that nonlinear counterion response generates not only effective many-body interactions, but also corrections to the effective pair and one-body interactions. It is these corrections [Eqs. (23) and (24)] whose impact on phase behavior we examine below in Sec. IV Note that the final terms on the right sides of Eqs. (22)-(24) originate from the charge neutrality condition, which required special treatment of the $k=0$ terms in Eqs. (16) and (20). A simple physical interpretation of microion response and its connection to microion-induced effective interactions between macroions is discussed in ref. [59].

\section{Random Phase Approximation}

Further progress requires specifying the OCP response functions. To this end, we note first that the counterions are usually characterized by relatively small electrostatic coupling parameters, $\Gamma=\lambda_{B} / a_{c} \ll 1$, where $\lambda_{B}=\beta z^{2} e^{2} / \epsilon$ is the Bjerrum length and $a_{c}=\left(3 / 4 \pi n_{c}\right)^{1 / 3}$ is the counterion-sphere radius. In such weakly-coupled plasmas, short-range correlations are often weak enough to justify a random phase approximation (RPA) 69], whereby the two-particle direct correlation function (DCF) is approximated by its exact asymptotic limit: $c^{(2)}(r)=-\beta v_{c c}(r)$ or $\hat{c}^{(2)}(k)=-4 \pi \beta z^{2} e^{2} / \epsilon k^{2}$. The OCP linear and first nonlinear response functions then take the simple analytical forms

$$
\chi(k)=\frac{-\beta n_{c}}{1+\kappa^{2} / k^{2}}
$$

and

$$
\chi^{\prime}\left(\mathbf{k}, \mathbf{k}^{\prime}\right)=-\frac{k_{B} T}{2 n_{c}^{2}} \chi(k) \chi\left(k^{\prime}\right) \chi\left(\left|\mathbf{k}+\mathbf{k}^{\prime}\right|\right),
$$

where $\kappa=\sqrt{4 \pi n_{c} z^{2} e^{2} / \epsilon k_{B} T}$ is the Debye screening constant (inverse screening length). Higher-order nonlinear response leads to higher-order terms in the effective Hamiltonian [Eq. (19)], which are here neglected.

Practical expressions for the effective interactions follow from specifying the macroion-counterion interaction inside the macroion core so as to minimize counterion penetration - a strategy similar to that of the pseudopotential theory of simple metals [71, 72]. The choice

$$
v_{m c}(r)=-\frac{Z z e^{2} \kappa}{\epsilon(1+\kappa a)}, \quad r<a,
$$

ensures zero counterion penetration $\left(\rho_{c}(r)=0, r<a\right)$ at the level of linear response [49, 57, 58] and virtually eliminates counterion penetration in the case of nonlinear response [59]. Substituting the Fourier transform of Eq. (27),

$$
\hat{v}_{m c}(k)=-\frac{4 \pi Z z e^{2}}{\epsilon(1+\kappa a) k^{2}}\left[\cos (k a)+\frac{\kappa}{k} \sin (k a)\right],
$$

into Eqs. (17), (18), and (22)-(24) then yields the effective interactions.

Upon reintroducing salt ions as a second species of microion [58], analytical expressions are obtained [59] for the volume energy and the effective pair potential. The volume energy is the sum of the linear response approximation

$$
E_{0}=F_{\text {plasma }}-N_{m} \frac{Z^{2} e^{2}}{2 \epsilon} \frac{\kappa}{1+\kappa a}-\frac{k_{B} T}{2} \frac{\left(N_{+}-N_{-}\right)^{2}}{N_{+}+N_{-}}
$$

and the first nonlinear correction

$$
\Delta E=\frac{N_{m} k_{B} T}{6} \frac{\left(n_{+}-n_{-}\right)}{n_{\mu}^{3}}\left[\frac{Z^{2} \kappa^{3} n_{\mu}}{8 \pi}\left(\frac{1}{1+\kappa a}\right)^{2}-\frac{Z^{3} \kappa^{6}}{(4 \pi)^{2}}\left(\frac{e^{\kappa a}}{1+\kappa a}\right)^{3} \mathrm{E}_{1}(3 \kappa a)\right],
$$

where $F_{\text {plasma }}=k_{B} T\left[N_{+} \ln \left(n_{+} \Lambda^{3}\right)+N_{-} \ln \left(n_{-} \Lambda^{3}\right)\right]$ is the ideal-gas free energy of the plasma, $n_{ \pm}=N_{ \pm} / V^{\prime}$ and $n_{\mu}=N_{\mu} / V^{\prime}=n_{+}+n_{-}=n_{c}+2 n_{s}$ are the microion number densities in the free volume, $\Lambda$ is the 
thermal wavelength of the microions, and

$$
\kappa=\left(\frac{4 \pi z^{2} e^{2} n_{\mu}}{\epsilon k_{B} T}\right)^{1 / 2}=\left(\frac{4 \pi z^{2} e^{2}}{\epsilon k_{B} T} \frac{\left(N_{c}+2 N_{s}\right)}{V(1-\eta)}\right)^{1 / 2}
$$

is the Debye screening constant, which depends on the total density of microions, adjusted for macroion excluded volume. The effective pair potential is the sum of

$$
v_{0}^{(2)}(r)=\frac{Z^{2} e^{2}}{\epsilon}\left(\frac{e^{\kappa a}}{1+\kappa a}\right)^{2} \frac{e^{-\kappa r}}{r}, \quad r>\sigma
$$

which is identical to the DLVO potential with a density-dependent screening constant, and

$$
\Delta v_{\mathrm{eff}}^{(2)}(r)=f_{1}(r) \frac{e^{-\kappa r}}{r}+f_{2}(r) \frac{e^{\kappa r}}{r}+f_{3}(r) \frac{e^{-\kappa a}}{r}, \quad r>\sigma
$$

where

$$
\begin{gathered}
f_{1}(r)=C_{1}\left[\kappa(r-\sigma)+1-e^{-\kappa \sigma}\right]+C_{2}\left[\mathrm{E}_{1}(\kappa(r-a))+\mathrm{E}_{1}(3 \kappa a)-\mathrm{E}_{1}(\kappa a)\right], \\
f_{2}(r)=-C_{2} \mathrm{E}_{1}(3 \kappa(r+a)), \\
f_{3}(r)=C_{2}\left[\mathrm{E}_{1}(2 \kappa(r+a))-\mathrm{E}_{1}(2 \kappa(r-a))\right], \\
C_{1}=\frac{1}{6} \frac{\left(n_{+}-n_{-}\right)}{n_{\mu}} \frac{Z^{2} e^{2}}{\epsilon}\left(\frac{e^{\kappa a}}{1+\kappa a}\right)^{2}, \\
C_{2}=\frac{1}{8 \pi} \frac{\left(n_{+}-n_{-}\right)}{n_{\mu}^{2}} \frac{Z^{3} e^{2} \kappa^{3}}{z \epsilon}\left(\frac{e^{\kappa a}}{1+\kappa a}\right)^{3},
\end{gathered}
$$

and

$$
\mathrm{E}_{1}(x)=\int_{1}^{\infty} \mathrm{d} u \frac{e^{-x u}}{u}, \quad x>0
$$

is the exponential integral function. The effective three-body interaction can be computed from the generalizations of Eqs. (18), (26), and (28), with the result [59]

$$
v_{\text {eff }}^{(3)}\left(\mathbf{r}_{1}-\mathbf{r}_{2}, \mathbf{r}_{1}-\mathbf{r}_{3}\right)=-k_{B} T \frac{\left(n_{+}-n_{-}\right)}{n_{\mu}^{3}} \int \mathrm{d} \mathbf{r} \rho_{1}\left(\left|\mathbf{r}_{1}-\mathbf{r}\right|\right) \rho_{1}\left(\left|\mathbf{r}_{2}-\mathbf{r}\right|\right) \rho_{1}\left(\left|\mathbf{r}_{3}-\mathbf{r}\right|\right),
$$

where

$$
\rho_{1}(r)= \begin{cases}\frac{Z}{z} \frac{\kappa^{2}}{4 \pi} \frac{e^{\kappa a}}{1+\kappa a} \frac{e^{-\kappa r}}{r}, & r>a, \\ 0, & r<a\end{cases}
$$

is the density of counterions around an isolated macroion.

It is important to establish the accuracy of the effective interactions predicted by the nonlinear response theory described above. In a direct comparison with ab initio simulations [76], first-order nonlinear corrections were shown to quantitatively match effective pair energies [59]. Nevertheless, the effective interactions predicted by response theory should be tested further, perhaps by comparisons with nonlinear PoissonBoltzmann theory. 


\section{B. Thermodynamic Phase Behavior}

\section{Variational Theory}

The effective electrostatic interactions predicted by response theory provide the basic input required by statistical mechanical theories and computer simulations of the effective one-component model of charged colloids. The one-component model is considerably simpler than the (multi-component) primitive model, and thus a practical alternative for investigating thermodynamic phase behavior and other bulk properties of many-particle systems. Here we input effective interparticle interactions into an approximate variational theory for the free energy. The Helmholtz free energy $F$ separates naturally into three contributions:

$$
F\left(T, V, N_{m}, N_{s}\right)=F_{\text {id }}\left(T, V, N_{m}\right)+F_{\text {ex }}\left(T, V, N_{m}, N_{s}\right)+E\left(T, V, N_{m}, N_{s}\right),
$$

where $F_{\text {id }}=N_{m} k_{B} T\left[\ln \left(n_{m} \Lambda_{m}^{3}\right)-1\right]$ is the exact ideal-gas free energy of a uniform fluid of macroions of thermal wavelength $\Lambda_{m}, F_{\text {ex }}$ is the excess free energy, which depends on effective intermacroion interactions, and $E$ is the one-body volume energy. Note that $F_{\text {ex }}$ and $E$ depend on the average densities of both macroions and salt ions.

To approximate the excess free energy, we apply a variational approach based on first-order thermodynamic perturbation theory, as in ref. [50]. Given a decomposition of the effective pair potential into reference and perturbation potentials,

$$
v_{\text {eff }}^{(2)}(r)=v_{\text {ref }}^{(2)}(r)+v_{\text {pert }}^{(2)}(r)
$$

an upper bound on the excess free energy density, $f_{\mathrm{ex}}=F_{\mathrm{ex}} / V$, is provided by the Gibbs-Bogoliubov inequality [69]

$$
f_{\text {ex }} \leq f_{\text {ref }}+\frac{1}{2} n_{m}^{2} \int \mathrm{d} \mathbf{r} g_{\text {ref }}(r) v_{\text {pert }}^{(2)}(r)
$$

where $f_{\text {ref }}$ and $g_{\text {ref }}(r)$ are the excess free energy density and radial distribution function, respectively, of the reference system. The short-range-repulsive form of the effective pair potential naturally suggests a hardsphere (HS) reference system. Thus, $v_{\text {ref }}^{(2)}(r)=v_{\mathrm{HS}}(r ; d)$, the pair potential between hard spheres of effective diameter $d$, and $v_{\text {pert }}^{(2)}(r)=v_{\text {eff }}^{(2)}(r), r \geq d$. The effective HS diameter provides a variational parameter with respect to which the right side of Eq. (44) can be minimized to impose a least upper bound on the excess free energy:

$$
f_{\mathrm{ex}}\left(n_{m}, n_{s}\right) \simeq \min _{(d)}\left\{f_{\mathrm{HS}}\left(n_{m}, n_{s} ; d\right)+2 \pi n_{m}^{2} \int_{d}^{\infty} \mathrm{d} r r^{2} g_{\mathrm{HS}}\left(r, n_{m} ; d\right) v_{\mathrm{eff}}^{(2)}\left(r, n_{m}, n_{s}\right)\right\} .
$$

Here $f_{\mathrm{HS}}\left(n_{m}, n_{s} ; d\right)$ and $g_{\mathrm{HS}}\left(r, n_{m} ; d\right)$ are, respectively, the excess free energy density and radial distribution function of the HS reference fluid, which we approximate by the essentially exact Carnahan-Starling and Verlet-Weis analytical expressions [69]. In practice, the exponential decay of $v_{\text {eff }}^{(2)}(r)$ with $r$ ensures rapid convergence of the perturbation integral in Eq. (45), justifying the further approximation that $g_{\mathrm{HS}}(r)=1$ for $r \geq 5 d$. The accuracy of the variational theory in predicting the equation of state has been confirmed by independent comparisons with Monte Carlo simulation data [50, 77].

\section{Grand Potential and Phase Coexistence}

For a system at fixed temperature, volume, and number of macroions, in osmotic equilibrium with a salt reservoir at fixed salt chemical potential $\mu_{s}$, the appropriate thermodynamic potential (minimized at equilibrium) is the semi-grand potential,

$$
\Omega\left(T, V, N_{m}, \mu_{s}\right)=F\left(T, V, N_{m}, N_{s}\right)-\mu_{s} N_{s}=-p V+\mu_{m} N_{m}
$$

where $p$ is the bulk pressure and $\mu_{m}$ is the chemical potential of the pseudomacroions. More precisely, $\mu_{m}$ is the change in free energy - at constant $T$ and $V$ - upon adding a bare macroion and its $Z / z$ neutralizing 
counterions and $\mu_{s}$ is the change in free energy upon adding a charge-neutral pair of salt ions. The semi-grand potential density is then given by

$$
\omega\left(T, n_{m}, \mu_{s}\right)=\Omega / V=f\left(T, n_{m}, n_{s}\right)-\mu_{s} n_{s}=-p+\mu_{m} n_{m},
$$

where $f=F / V$ is the total free energy density and $n_{s}=N_{s} / V$ is the number density of salt ion pairs in the system. At constant $T$, the differential relation

$$
\mathrm{d} \Omega\left(T, V, N_{m}, \mu_{s}\right)=-p \mathrm{~d} V+\mu_{m} \mathrm{~d} N_{m}-N_{s} \mathrm{~d} \mu_{s},
$$

yields the pressure

$$
p=-\left(\frac{\partial \Omega}{\partial V}\right)_{T, N_{m}, \mu_{s}}=n_{m}\left(\frac{\partial \omega}{\partial n_{m}}\right)_{T, \mu_{s}}-\omega
$$

and the macroion chemical potential

$$
\mu_{m}=\left(\frac{\partial \Omega}{\partial N_{m}}\right)_{T, V, \mu_{s}}=\left(\frac{\partial \omega}{\partial n_{m}}\right)_{T, \mu_{s}} .
$$

Equilibrium coexistence of bulk phases requires equality of pressure and of chemical potentials (of macroions and salt ions) in the two phases (1 and 2):

$$
\begin{aligned}
& p^{(1)}=p^{(2)} \\
& \mu_{m}^{(1)}=\mu_{m}^{(2)} \\
& \mu_{s}^{(1)}=\mu_{s}^{(2)}=\mu_{s}^{(r)},
\end{aligned}
$$

where the superscript $(r)$ denotes a reservoir quantity. Equality of pressure is equivalent to equality of osmotic pressure, $\Pi=p-p^{(r)}$, i.e., the difference between the system and reservoir pressures. The osmotic pressure - a manifestation of the Donnan effect [1] - vanishes in the dilute limit of zero colloid concentration.

The coexistence conditions have simple geometrical interpretations. Equations (47)-(53) describe a common tangent, of slope $\mu_{m}$ and intercept $-p$, to the curve of $\omega\left(n_{m}, \mu_{s}\right)$ vs. $n_{m}$ (constant $\mu_{s}$ ), or equivalently a Maxwell equal-area construction. Specifically, the relations

$$
\int_{1}^{2} \mathrm{~d} \omega=\int_{1}^{2} \mathrm{~d} n_{m} \mu_{m}\left(n_{m}, \mu_{s}\right)=\mu_{m}^{(1)}\left(n_{m}^{(2)}-n_{m}^{(1)}\right)
$$

and

$$
\int_{1}^{2} \mathrm{~d}\left(\Omega / N_{m}\right)=-\int_{1}^{2} \mathrm{~d} v_{m} p\left(v_{m}, \mu_{s}\right)=-p^{(1)}\left(v_{m}^{(2)}-v_{m}^{(1)}\right),
$$

with $v_{m}=V / N_{m}=1 / n_{m}$, imply that constant- $\mu_{s}$ curves of $\mu_{m}\left(n_{m}, \mu_{s}\right)$ vs. $n_{m}$ and of $p\left(v_{m}, \mu_{s}\right)$ vs. $v_{m}$ enclose equal areas above and below the horizontal lines $\mu_{m}=\mu_{m}^{(1)}=\mu_{m}^{(2)}$ and $p=p^{(1)}=p^{(2)}$, respectively. Changes of curvature sufficient to allow common-tangent constructions on the semi-grand potential, and equal-area constructions on the chemical potential and pressure, imply phase coexistence.

At low salt concentrations, the salt reservoir behaves as an ideal gas of ions, whose pressure and chemical potential are well approximated by

$$
p^{(r)}=2 n_{s}^{(r)} k_{B} T
$$

and

$$
\mu_{s}^{(r)}=2 k_{B} T \ln \left(n_{s}^{(r)} \Lambda^{3}\right),
$$

where $n_{s}^{(r)}$ is the reservoir number density of pairs of salt ions of thermal wavelength $\Lambda$. Note that $\Lambda$ and $\Lambda_{m}$ are arbitrary, as they contribute to the semi-grand potential only terms that are linear in density, which do not affect the coexisting densities. 
The phase diagram is computed as follows. For a given macroion density $n_{m}$ and salt chemical potential (i.e., reservoir salt density $n_{s}^{(r)}$ ), the system salt density $n_{s}$ is numerically determined [from Eq. (53)] as the solution of

$$
\mu_{s}=\left(\frac{\partial f\left(n_{m}, n_{s}\right)}{\partial n_{s}}\right)_{T, n_{m}}=2 k_{B} T \ln \left(n_{s}^{(r)} \Lambda^{3}\right)
$$

where $f$ is the total free energy density, the excess part of which is given by Eq. (45). In the case of linear response, Eq. (158) can be expressed in a somewhat more practical form by separating out and analytically evaluating the dominant volume energy contribution. Substituting Eqs. (29) and (42) into Eq. (58) then yields

$$
\beta \mu_{s}=\ln \left[\left(n_{c}+n_{s}\right) \Lambda^{3}\right]+\ln \left(n_{s} \Lambda^{3}\right)-\frac{Z \kappa \lambda_{B}}{2(1+\kappa a)^{2}} \frac{n_{c}}{n_{\mu}}+\left(\frac{n_{c}}{n_{\mu}}\right)^{2}+\beta\left(\frac{\partial f_{\mathrm{ex}}\left(n_{m}, n_{s}\right)}{\partial n_{s}}\right)_{n_{m}} .
$$

The pressure and macroion chemical potential are next computed from Eqs. (49) and (50). Finally, the macroion and salt densities are varied to satisfy the remaining coexistence conditions [Eqs. (51) and (52)].

\section{RESULTS AND DISCUSSION}

To investigate the influence of nonlinear microion screening on the phase behavior of deionized charged colloids, the variational theory (Sec. IIIB) is used to compute the semi-grand potential, taking as input the effective interactions predicted by response theory (Sec. IIIA). By performing a coexistence analysis and comparing the phase diagrams that result from linear and first-order nonlinear interactions, leading-order nonlinear effects are quantified. For simplicity, effective three-body interactions are here neglected, since these are always attractive [59] and thus would only promote phase separation. In this way, we isolate the main nonlinear corrections to the volume energy and effective pair potential and assess their impact on phase behavior.

Numerical results are presented for the case of room-temperature aqueous suspensions $\left(\lambda_{B}=0.72 \mathrm{~nm}\right)$ and monovalent counterions $(z=1)$. For several choices of macroion radius $a$, the effective macroion valence $Z$ is set near the threshold for charge renormalization 73], $Z \sim O(10)\left(a / \lambda_{B}\right)$. Figure 2 illustrates the effective pair and triplet potentials vs. macroion separation, with linear and nonlinear screening, for various sets of system parameters. The particular case of $(\sigma=266 \mathrm{~nm}, Z=1217)$ is included to permit direct comparison with ref. [50]. While nonlinear screening generally softens repulsive pair interactions, the correction is relatively minor for the selected macroion diameters and valences. The effective triplet potential, shown for an equilateral triangle arrangement of three macroions, is always attractive and decays rapidly with increasing separation. In passing, we note that the triplet interactions that arise within response theory [59] differ in definition from their counterparts in Poisson-Boltzmann theory [74, 75].

Figures $[3$ and 4 present predictions for the osmotic pressure $\Pi$ (equation of state) vs. volume fraction $\eta$ at fixed reservoir salt concentration $c_{s}^{(r)}$ (or salt chemical potential $\mu_{s}$ ). The variation of $\Pi$ with $\eta$ is a diagnostic of thermodynamic stability, a negative slope signaling instability toward phase separation (see below). Figure 3 illustrates that, within the linearized theory, the system becomes unstable below a certain critical salt concentration. Figure 4 demonstrates the sensitivity of the osmotic pressure to nonlinear screening, which originates mainly from the nonlinear correction to the volume energy.

Figures [5] and [6] present the corresponding system salt concentration $c_{s}$ (in $\mu$ mol/liter) vs. volume fraction (at fixed $\mu_{s}$ ). The monotonic decrease of $c_{s}$ with increasing $\eta$ follows from Eq. (59) and stems from an interplay between salt entropy and salt-macroion interactions. Entropy and excluded-volume interactions alone would give a simple linear decline, $c_{s}=(1-\eta) c_{s}^{(r)}$, with a slope of $-c_{s}^{(r)}$. However, salt-macroion electrostatic interactions tend to expel salt from the system, steepening the decline, while maintaining an approximate linear dependence over a considerable range of $\eta$. As illustrated in Fig. 6] nonlinear screening, which modifies the state dependence of the effective interactions, tends to lower the system salt concentration.

Figure [ typifies the monotonic decrease of the effective hard-sphere diameter $d$, and increase of the Debye screening constant $\kappa$, with increasing volume fraction at fixed $\mu_{s}$. Nonlinear screening evidently reduces both $d$ and $\kappa$. For the chosen parameters, the reduction appears modest, but is significant, given the sensitivity of the free energy to these parameters. 
Figures [3] 4] and 8] illustrate that, for sufficiently high macroion valence and low salt concentration, van der Waals loops emerge in the equation of state at fixed $\mu_{s}$ - a direct signature of phase instability. The maximimum and minimum in the curve of osmotic pressure vs. volume fraction mark the vapor and liquid spinodal densities, respectively, between which the compressibility is negative and the uniform fluid is unstable with respect to phase separation [Fig. 8(a)]. Correspondingly, an equal-area construction on the curve of osmotic pressure vs. inverse volume fraction [Fig. 8 (b)], or of chemical potential vs. volume fraction [Fig. 8(c)], yields the densities of the coexisting vapor and liquid phases. A scan over reservoir salt concentration (salt chemical potential) traces out the spinodal and binodal (coexistence) curves in the phase diagram.

Figure 9 presents the resulting fluid phase diagrams for highly deionized suspensions as predicted by variational theory with both linear and nonlinear effective interactions as input. In each case, above a critical salt concentration, the uniform fluid is thermodynamically stable. Below the critical point, the fluid separates into macroion-rich (liquid) and macroion-poor (vapor) bulk phases, the salt concentration playing a role analogous to temperature in the liquid-vapor separation of a simple one-component fluid. For the parameter regime investigated here, the density of the liquid phase is found to be always well below the threshold for freezing, estimated from the hard-sphere freezing criterion, $\eta(d / \sigma)^{3} \sim 0.49$, with the charged colloids approximated as neutral hard spheres of effective diameter $d$.

The tie lines in the phase diagrams of Fig. 9 join corresponding points on the liquid and vapor binodals (and spinodals) and, if extended, intersect the $\eta=0$ axis at the respective reservoir salt concentrations. The fact that the tie lines all have essentially the same slope, independent of reservoir salt concentration, is a physical consequence of strong salt-macroion electrostatic interactions, as described by Eq. (59). The influence of nonlinear response on the tie-line slopes is negligible for the parameters here investigated.

The predicted phase separation of charged colloids is remarkable, considering that simple one-component systems, interacting via purely repulsive pair potentials, exhibit only a single fluid phase. Within the present theoretical framework, phase instability at low salt concentrations is driven by the strong density dependence of the effective interactions, chiefly the one-body volume energy in deionized suspensions. It should be emphasized that because the colloid and salt concentrations vary between the two phases, the density-dependent effective interactions also differ in the two phases.

The unusual phase separation can be understood, more fundamentally, as the result of a classic competition between entropy and energy. On one side of the balance, favoring a stable uniform fluid, are the configurational entropies of all ions, represented by the ideal-gas terms in Eqs. (29) and (42), and the positive potential energy of macroion pair repulsion. On the other side is the (density-dependent) negative potential energy of macroion-counterion attraction [second term on the right side of Eq. (29)], which favors a concentrated phase with counterions localized around, and thus strongly attracted to, the macroions.

Within the "entropy vs. energy" view, the sensitivity of phase behavior to salt concentration becomes clearer. At salt concentrations low enough that screening is counterion-dominated and screening lengths are relatively long, the counterion distribution is so diffuse that counterion-macroion attraction is too weak to drive macroion aggregation. With increasing salt concentration, the screening length shortens, the counterions become more localized around the macroions, and counterion-macroion attraction may - for sufficiently high macroion valence - overcome configurational entropy and macroion pair repulsion to drive phase separation. The resulting concentrated phase is energetically favored, the counterions being closer on average to the macroions, but entropically disfavored, since the microions (excluded by macroion cores) must occupy a smaller free volume. On the other hand, the dilute phase is energetically disfavored, the counterions tending to roam farther from the macroions, but is entropically favored, since the microions can explore a larger free volume. At salt concentrations high enough that screening is salt-dominated, the salt-ion entropy overwhelms the counterion-macroion interaction energy in the free energy and prevents macroion aggregation.

Thermodynamic phase behavior qualitatively similar to that depicted in Fig. 9] has been predicted before [50, 54]. Compared with the results of van Roij et al. 50], based on essentially the same variational theory for free energies, but a linearized density-functional theory for effective interactions, the present theory predicts a somewhat larger unstable area in the phase diagram. This quantitative discrepancy results mainly from different treatments of excluded-volume effects in the two approaches. In particular, the excluded-volume correction to the screening constant in response theory $[1 /(1-\eta)$ factor in Eq. (31)] enhances microion screening and promotes phase instability. 


\section{SUMMARY AND CONCLUSIONS}

In summary, we have investigated the controversial issue of phase separation in deionized charge-stabilized colloidal suspensions by inputting effective electrostatic interactions from response theory into free energies from a thermodynamic variational theory. By considering both linear and first-order nonlinear approximations for the effective pair potential and one-body volume energy, we have systematically assessed the influence of nonlinear screening on phase behavior. A coexistence analysis results in osmotic pressures [Figs. 3] 4] and 8] and phase diagrams [Fig. 9] that clearly exhibit thermodynamic instability towards phase separation for sufficiently high macroion effective valences and low salt concentrations.

For macroion sizes and effective valences within limits established by charge renormalization considerations, first-order nonlinear corrections to the effective interactions are relatively weak and can either enhance or diminish stability of the uniform fluid phase, depending on system parameters. In general, the higher the macroion surface charge density, the higher the critical salt concentration and the larger the area of the unstable region in the phase diagram. Our main conclusion is that, within the present model, nonlinear screening appears not to suppress phase separation of deionized suspensions, contradicting conclusions drawn from previous studies [60, 61, 62, 63] and raising hope that a similar phenomenon may yet be observed in simulations of the primitive model.

In closing, three key approximations of the present approach deserve to be highlighted for further scrutiny. First, the neglect of higher-order nonlinear corrections to the effective interactions presumes that nonlinear effects are strongest at the one- and two-body levels. The finding that first-order nonlinear corrections do not qualitatively alter fluid phase behavior suggests that higher-order corrections are unlikely to have drastic consequences - for example, suppression of phase separation. Furthermore, the presumption of weak manybody effective interactions is consistent with the dominance of the volume energy in effective one-component models of simple metals [78, 79, 80, 81, 82], but should be further checked for charged colloids. Second, the mean-field approximation for the response functions of the microion plasma assumes weakly correlated microions. Although usually considered reasonable for monovalent microions, this assumption can and should be checked by more accurately modeling the structure of the microion plasma. Finally, the assumption of fixed macroion valence neglects the dependence of the effective valence on colloid and salt densities. This interesting issue of coupling between the effective macroion charge and phase behavior is being examined by means of charge renormalization theory and will be the subject of a future paper.

\section{Acknowledgments}

This work was supported by the National Science Foundation under Grant Nos. DMR-0204020 and EPS0132289 .

[1] R. J. Hunter, Foundations of Colloid Science (Oxford University Press, Oxford, 1986).

[2] P. N. Pusey, in Liquids, Freezing and Glass Transition, session 51, ed. J.-P. Hansen, D. Levesque, and J. ZinnJustin (North-Holland, Amsterdam, 1991).

[3] K. S. Schmitz, Macroions in Solution and Colloidal Suspension (VCH, New York, 1993).

[4] B. V. R. Tata, M. Rajalakshmi, and A. K. Arora, Phys. Rev. Lett. 69, 3778 (1992).

[5] K. Ito, H. Yoshida, and N. Ise, Science 263, 66 (1994).

[6] N. Ise and H. Yoshida, Acc. Chem. Res. 29, 3 (1996).

[7] B. V. R. Tata, E. Yamahara, P. V. Rajamani, and N. Ise, Phys. Rev. Lett. 78, 2660 (1997).

[8] N. Ise, T. Konishi, and B. V. R. Tata, Langmuir 15, 4176 (1999).

[9] H. Matsuoka, T. Harada, and H. Yamaoka, Langmuir 10, 4423(1994); H. Matsuoka, T. Harada, K. Kago, and H. Yamaoka, ibid 12, 5588 (1996); T. Harada, H. Matsuoka, T. Ikeda, and H. Yamaoka, ibid 15, 573 (1999).

[10] F. Gröhn and M. Antonietti, Macromolecules 33, 5938 (2000).

[11] A. E. Larsen and D. G. Grier, Nature 385, 230 (1997).

[12] J. Israelachvili, Intermolecular and Surface Forces (Academic, London, 1992).

[13] B. V. Derjaguin and L. Landau, Acta Physicochimica (USSR) 14, 633 (1941).

[14] E. J. W. Verwey and J. T. G. Overbeek, Theory of the Stability of Lyophobic Colloids (Elsevier, Amsterdam, 1948). 
[15] T. Palberg and M. Würth, Phys. Rev. Lett. 72, 786 (1994); B. V. R. Tata and A. K. Arora, Phys. Rev. Lett. 72, 787 (1994).

[16] L. Belloni, J. Phys.: Condens. Matter 12, R549 (2000).

[17] C. P. Royall, M. E. Leunissen, and A. van Blaaderen, J. Phys.: Condens. Matter 15, S3581 (2003).

[18] K. S. Schmitz, Acc. Chem. Res. 29, 7 (1996).

[19] K. S. Schmitz, Phys. Chem. Chem. Phys. 1, 2109 (1999).

[20] K. S. Schmitz, L. B. Bhuiyan, and A. K. Mukherjee, Langmuir 19, 7160 (2003).

[21] A. E. Larsen and D. G. Grier, Phys. Rev. Lett. 76, 3862 (1996).

[22] J. C. Crocker and D. G. Grier, Phys. Rev. Lett. 77, 1897 (1996).

[23] G. M. Kepler and S. Fraden, Phys. Rev. Lett. 73, 356 (1994).

[24] J. Baumgartl and C. Bechinger, Europhys. Lett. 71, 487-493 (2005); J. Baumgartl, J. L. Arauz-Lara, and C. Bechinger, preprint (cond-mat/0503105).

[25] J. C. Neu, Phys. Rev. Lett. 82, 1072 (1999).

[26] J. E. Sader and D. Y. C. Chan, J. Coll. Int. Sci. 213, 268 (1999); Langmuir 16, 324 (2000).

[27] E. Trizac and J. L. Raimbault, Phys. Rev. E 60, 6530 (1999); E. Trizac, Phys. Rev. E 62, R1465 (2000).

[28] B. Svensson and B. Jönsson, Chem. Phys. Lett. 108, 580 (1984).

[29] L. Guldbrand, B. Jönsson, H. Wennerström, and P. Linse, J. Chem. Phys. 80, 2221 (1984).

[30] R. Kjellander and S. Marčelja, Chem. Phys. Lett. 112, 49 (1984).

[31] R. Kjellander and S. Marčelja, J. Chem. Phys. 88, 7138 (1988).

[32] I. Rouzina and V. A. Bloomfield, J. Phys. Chem. 100, 9977 (1996).

[33] B.-Y. Ha and A. J. Liu, Phys. Rev. Lett. 79, 1289 (1997); Phys. Rev. E 58, 6281 (1998); ibid 60, 803 (1999).

[34] Y. Levin, J. J. Arenzon, and J. F. Stilck, Phys. Rev. Lett. 83, 2680 (1999); B.-Y. Ha and A. J. Liu, ibid 83, 2681 (1999).

[35] Y. Levin, Physica A 265, 432 (1999);

[36] M. J. Stevens, Phys. Rev. Lett. 82, 101 (1999); Biophys. J. 80, 130 (2001).

[37] F. J. Solis and M. Olvera de la Cruz, Phys. Rev. E 60, 4496 (1999).

[38] B. I. Shklovskii, Phys. Rev. E 60, 5802 (1999); T. T. Nguyen, I. Rouzina, and B. I. Shklovskii, Phys. Rev. E 60, 7032 (1999).

[39] T. T. Nguyen, I. Rouzina, and B. I. Shklovskii, J. Chem. Phys. 112, 2562 (2000); T. T. Nguyen, A. Yu. Grosberg, and B. I. Shklovskii, J. Chem. Phys. 113, 1110 (2000).

[40] A. Diehl, H. A. Carmona, and Y. Levin, Phys. Rev. E 64, 11804 (2001).

[41] W. M. Gelbart, R. F. Bruinsma, P. A. Pincus, and V. A. Parsegian, Physics Today 53, 38 (Sept. 2000).

[42] V. Lobaskin and P. Linse, J. Chem. Phys. 111, 4300 (1999); P. Linse and V. Lobaskin, Phys. Rev. Lett. 83, 4208 (1999); P. Linse, J. Chem. Phys. 113, 4359 (2000); J. Reščič and P. Linse, J. Chem. Phys. 114, 10131 (2001); V. Lobaskin, A. Lyubartsev, and P. Linse, Phys. Rev. E 63, 020401(R) (2001); V. Lobaskin and K. Qamhieh, J. Phys. Chem. B 107, 8022 (2003).

[43] A.-P. Hynninen, M. Dijkstra, and A. Z. Panagiotopoulos, J. Chem. Phys. 123, 084903 (2005).

[44] E. Allahyarov, I. D'Amico, and H. Löwen, Phys. Rev. Lett. 81, 1334 (1998).

[45] R. Messina, C. Holm, and K. Kremer, Phys. Rev. Lett. 85, 872 (2000); Eur. Phys. J. E 4, 363 (2001).

[46] J.-P. Hansen and H. Löwen, Ann. Rev. Phys. Chem. 51, 209 (2000).

[47] C. N. Likos, Phys. Rep. 348, 267 (2001).

[48] Y. Levin, Rep. Prog. Phys. 65, 1577 (2002).

[49] R. van Roij and J.-P. Hansen, Phys. Rev. Lett. 79, 3082 (1997).

[50] R. van Roij, M. Dijkstra, and J.-P. Hansen, Phys. Rev. E 59, 2010 (1999).

[51] R. van Roij and R. Evans, J. Phys.: Condens. Matter 11, 10047 (1999).

[52] B. Zoetekouw and R. van Roij, Phys. Rev. E 73, 021403 (2006).

[53] H. Graf and H. Löwen, Phys. Rev. E 57, 5744 (1998).

[54] P. B. Warren, J. Chem. Phys. 112, 4683 (2000); J. Phys.: Condens. Matter 15, S3467 (2003); preprint (cond-mat/0506537).

[55] B. Beresford-Smith, D. Y. C. Chan, and D. J. Mitchell, J. Coll. Int. Sci. 105, 216 (1985).

[56] D. Y. C. Chan, P. Linse, and S. N. Petris, Langmuir 17, 4202 (2001).

[57] A. R. Denton, J. Phys.: Condens. Matter 11, 10061 (1999).

[58] A. R. Denton, Phys. Rev. E 62, 3855 (2000).

[59] A. R. Denton, Phys. Rev. E 70, 31404 (2004).

[60] H. H. von Grünberg, R. van Roij, and G. Klein Europhys. Lett. 55, 580 (2001).

[61] M. Deserno and H. H. von Grünberg, Phys. Rev. E 66, 011401 (2002).

[62] M. N. Tamashiro and H. Schiessel, J. Chem. Phys. 119, 1855 (2003).

[63] Y. Levin, E. Trizac, and L. Bocquet, J. Phys.: Condens. Matter 15, S3523 (2003).

[64] M. E. Fisher, J. Stat. Phys. 75, 1 (1994); X. Li, Y. Levin, and M. E. Fisher, Europhys. Lett. 26, 683 (1994); M. E. Fisher, Y. Levin, and X. Li, J. Chem. Phys. 101, 2273 (1994).

[65] N. V. Sushkin and G. D. J. Phillies, J. Chem. Phys. 103, 4600 (1995). 
[66] L. E. González, D. J. González, M. Silbert, and S. Baer, Mol. Phys. 99, 875 (2001).

[67] J. S. Rowlinson, Mol. Phys. 52, 567 (1984).

[68] M. J. Grimson and M. Silbert, Mol. Phys. 74, 397 (1991).

[69] J.-P. Hansen and I. R. McDonald, Theory of Simple Liquids, $2^{\text {nd }}$ ed. (Academic, London, 1986).

[70] N. W. Ashcroft and D. Stroud, Solid State Phys. 33, 1 (1978).

[71] J. Hafner, From Hamiltonians to Phase Diagrams (Springer, Berlin, 1987).

[72] N. W. Ashcroft, Phys. Lett. 23, 48 (1966).

[73] S. Alexander, P. M. Chaikin, P. Grant, G. J. Morales, and P. Pincus, J. Chem. Phys. 80, 5776 (1984).

[74] C. Russ, H. H. von Grünberg, M. Dijkstra, R. van Roij, Phys. Rev. E 66, 011402 (2002).

[75] A.-P. Hynninen, M. Dijkstra, and R. van Roij, Phys. Rev. E 69, 61407 (2004).

[76] R. Tehver, F. Ancilotto, F. Toigo, J. Koplik, and J. R. Banavar, Phys. Rev. E 59, R1335 (1999).

[77] B. Lu and A. R. Denton, unpublished.

[78] C. J. Pethick, Phys. Rev. B 2, 1789 (1970).

[79] E. G. Brovman and G. Solt, Solid State Comm. 8, 903 (1970).

[80] S. P. Singh and W. H. Young, J. Phys. F: Metal Phys. 3, 1127 (1973).

[81] M. Rasolt and R. Taylor, Phys. Rev. B 11, 2717 (1975); L. Dagens, M. Rasolt, and R. Taylor, Phys. Rev. B 11, 2726 (1975).

[82] A. A. Louis and N. W. Ashcroft, Phys. Rev. Lett. 81, 4456 (1998). 


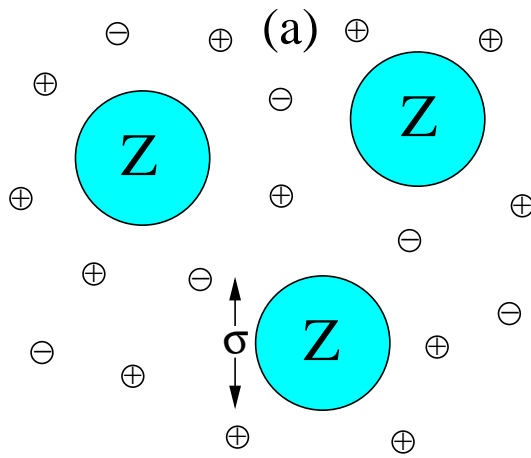

(b)
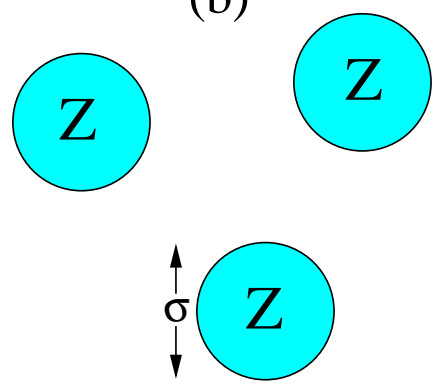

FIG. 1: Models of charge-stabilized colloidal suspensions: (a) Primitive model of charged hard-sphere macroions, of effective valence $Z$ and diameter $\sigma$, and microions (counterions, salt ions) suspended in a dielectric continuum. (b) Effective one-component model of pseudomacroions governed by effective interactions. 

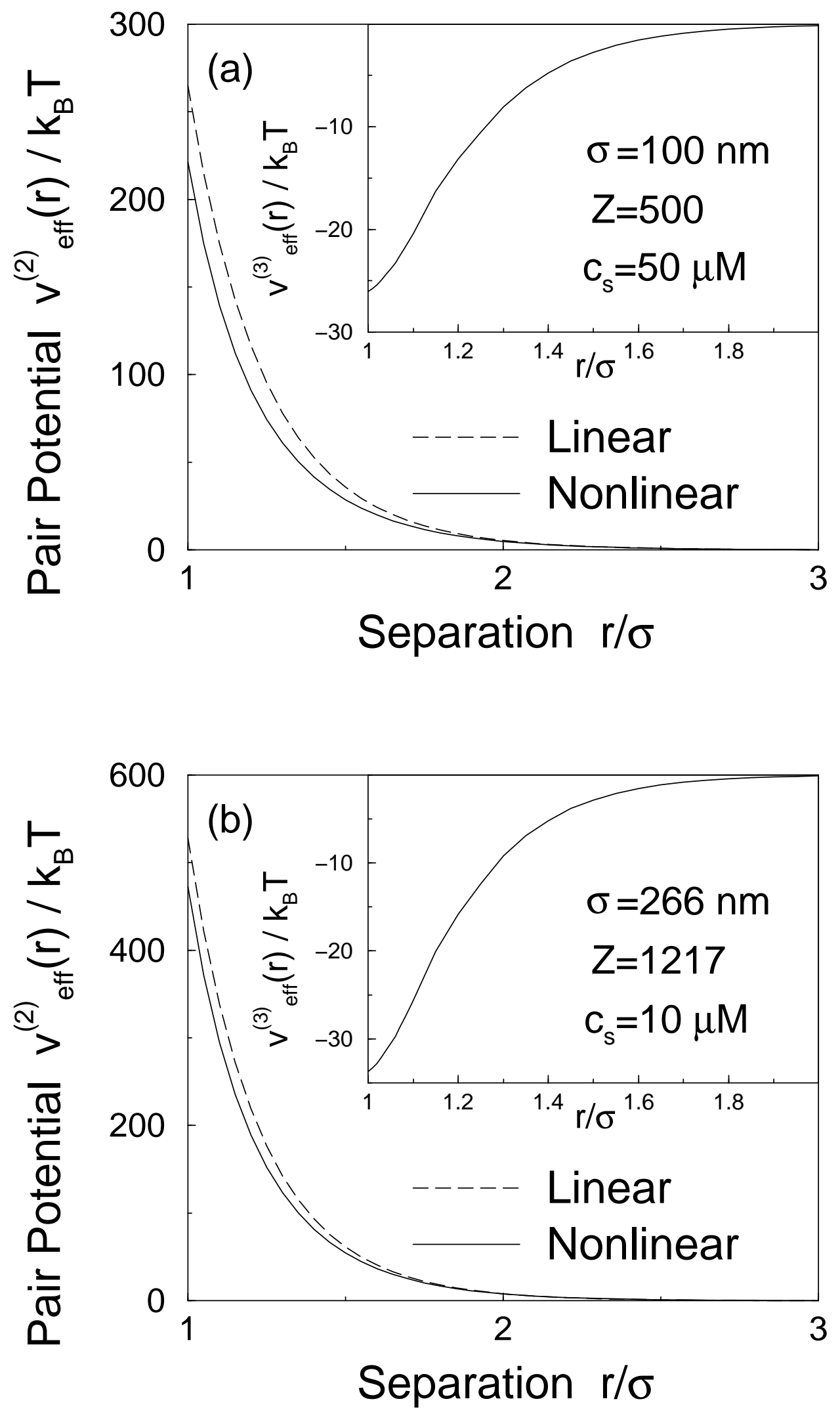


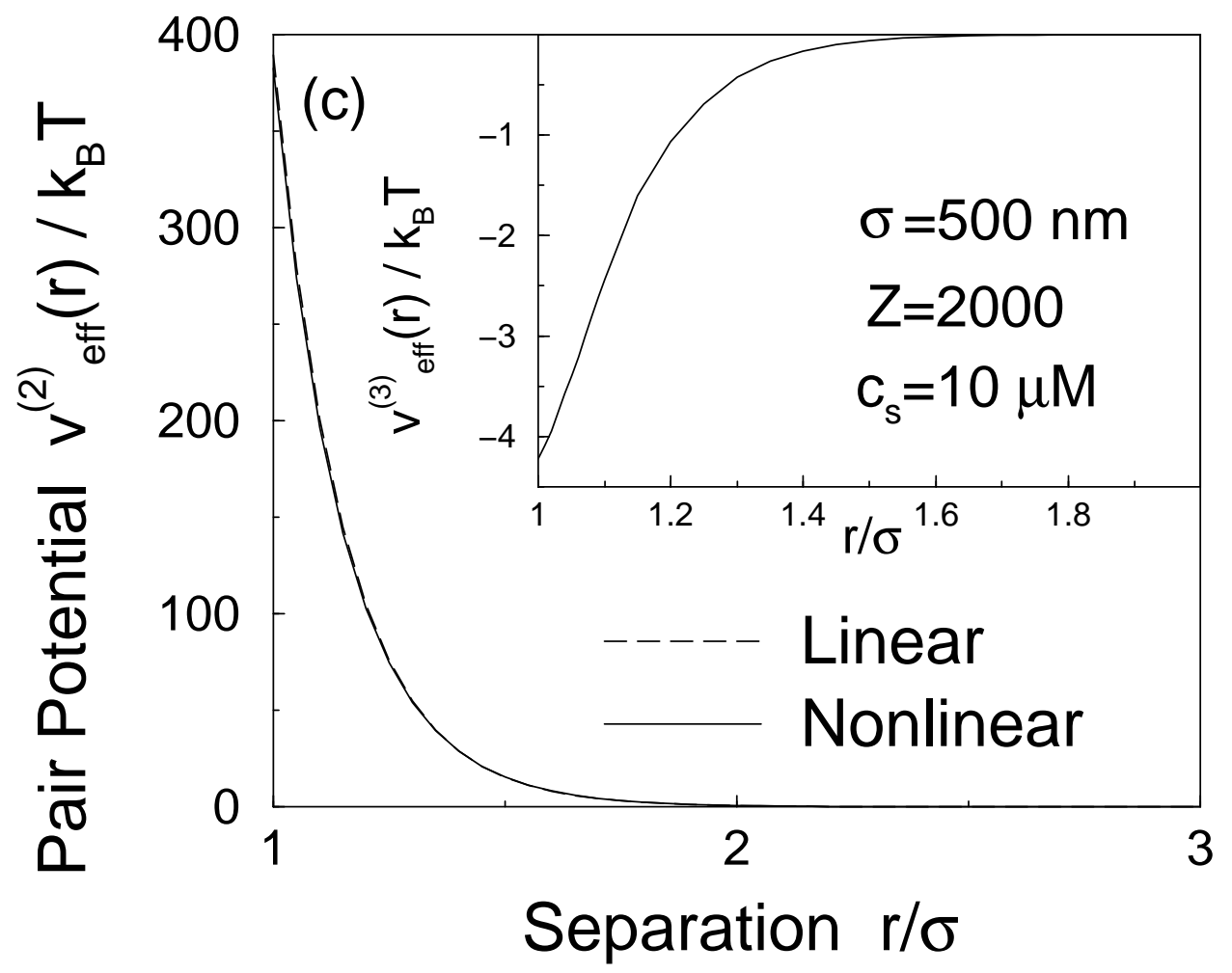

FIG. 2: Effective pair potential $v_{\text {eff }}^{(2)}(r)$ vs. center-to-center separation $r$ for fixed colloid volume fraction $\eta=0.05$ and various combinations of macroion diameter $\sigma$, effective valence $Z$, and system salt concentration $c_{s}$ : (a) $\sigma=100$ $\mathrm{nm}, Z=500, c_{s}=50 \mu \mathrm{M}$; (b) $\sigma=266 \mathrm{~nm}, Z=1217, c_{s}=10 \mu \mathrm{M}$; (c) $\sigma=500 \mathrm{~nm}, Z=2000, c_{s}=10 \mu \mathrm{M}$. Solid (dashed) curves are predictions of nonlinear (linear) response theory. Insets show corresponding effective triplet potentials $v_{\text {eff }}^{(3)}(r)$ for three macroions arranged in an equilateral triangle of side length $r$. 

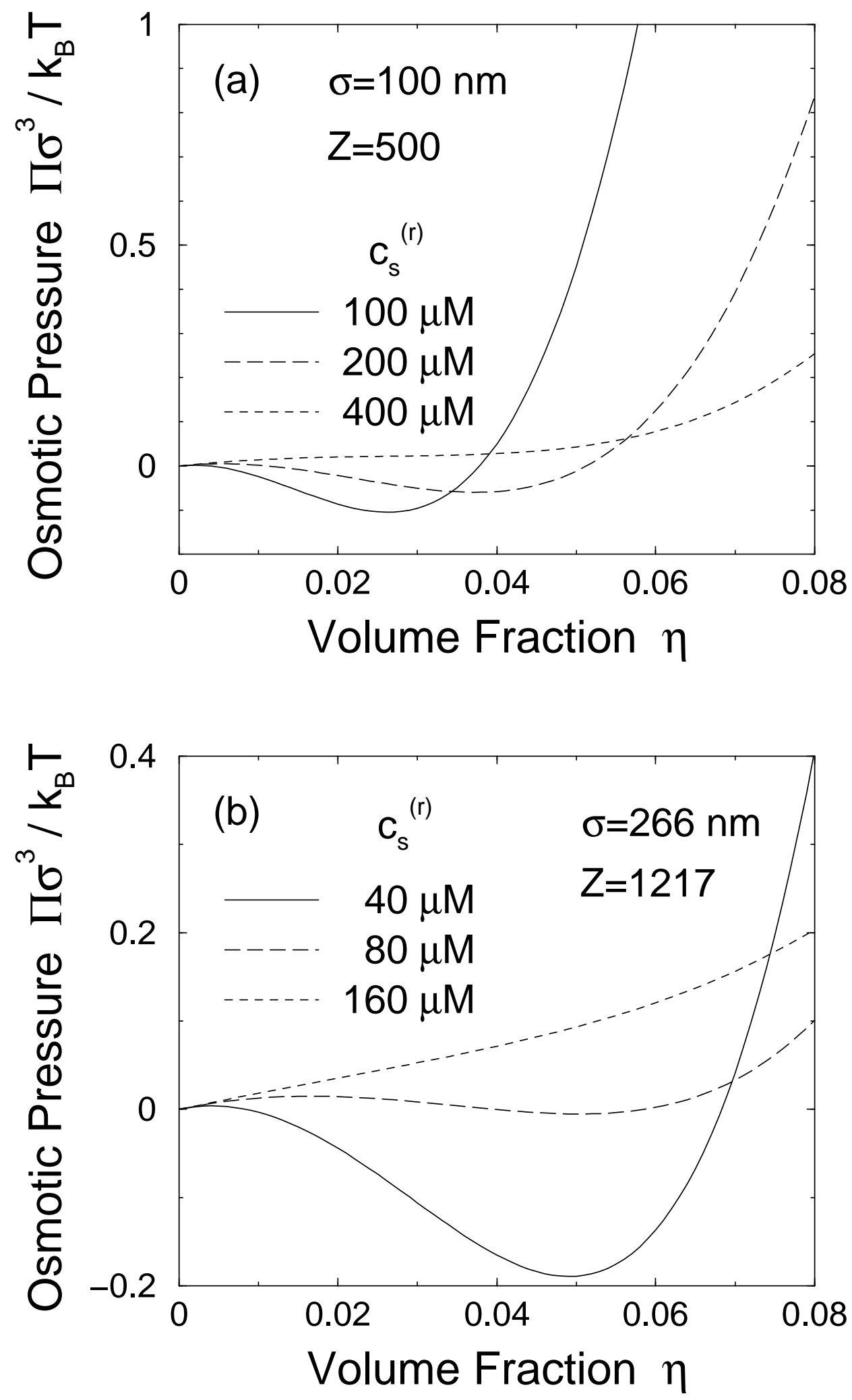


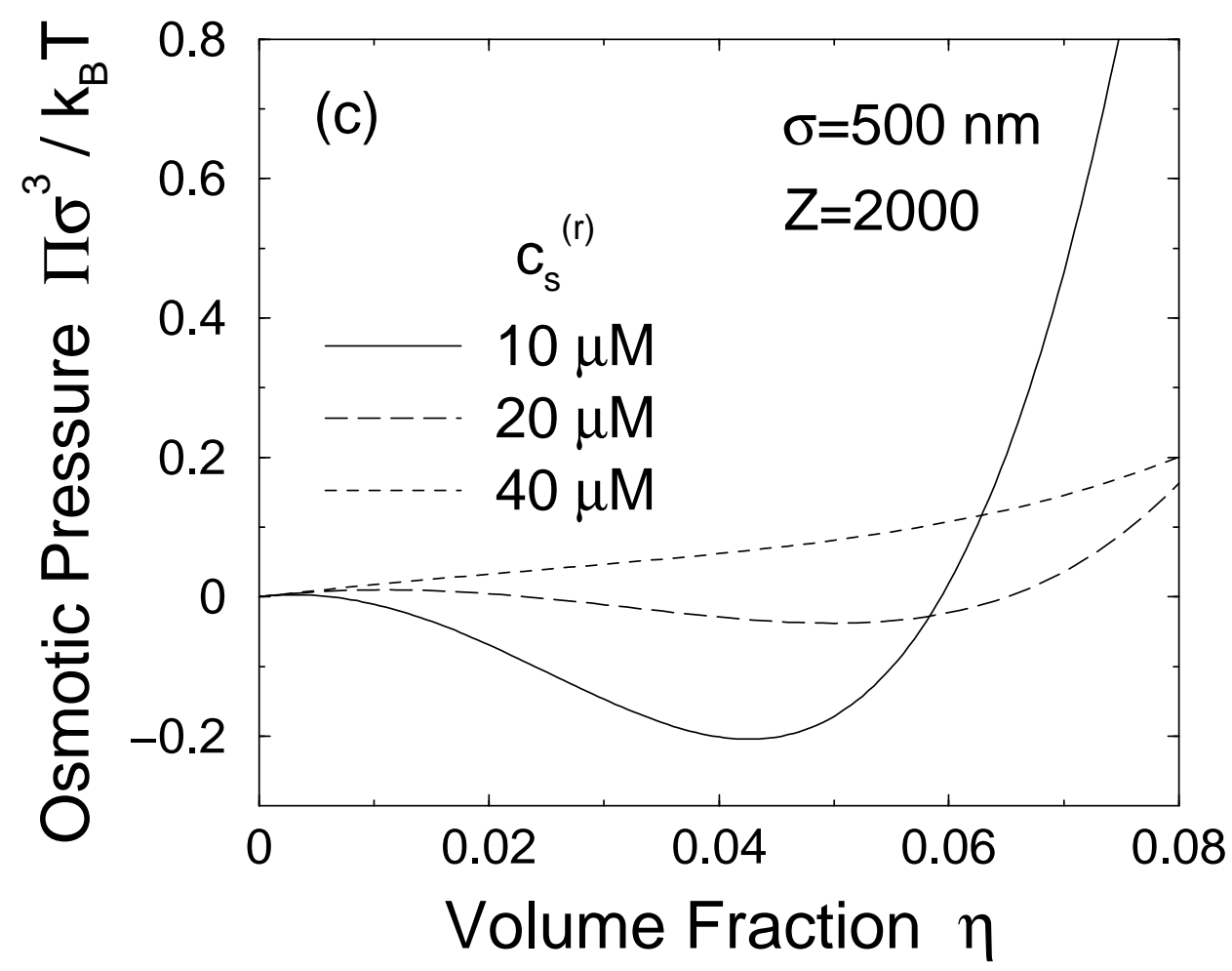

FIG. 3: Linear-screening predictions for osmotic pressure $\Pi$ (in reduced units) vs. colloid volume fraction $\eta$ for same combinations of macroion diameter $\sigma$ and valence $Z$ as in Fig. 2 and various fixed reservoir salt concentrations $c_{s}^{(r)}$ : (a) $\sigma=100 \mathrm{~nm}, Z=500, c_{s}^{(r)}=100,200,400 \mu \mathrm{M}$; (b) $\sigma=266 \mathrm{~nm}, Z=1217, c_{s}^{(r)}=40,80,160 \mu \mathrm{M}$; (c) $\sigma=500 \mathrm{~nm}$, $Z=2000, c_{s}^{(r)}=10,20,40 \mu \mathrm{M}$. 

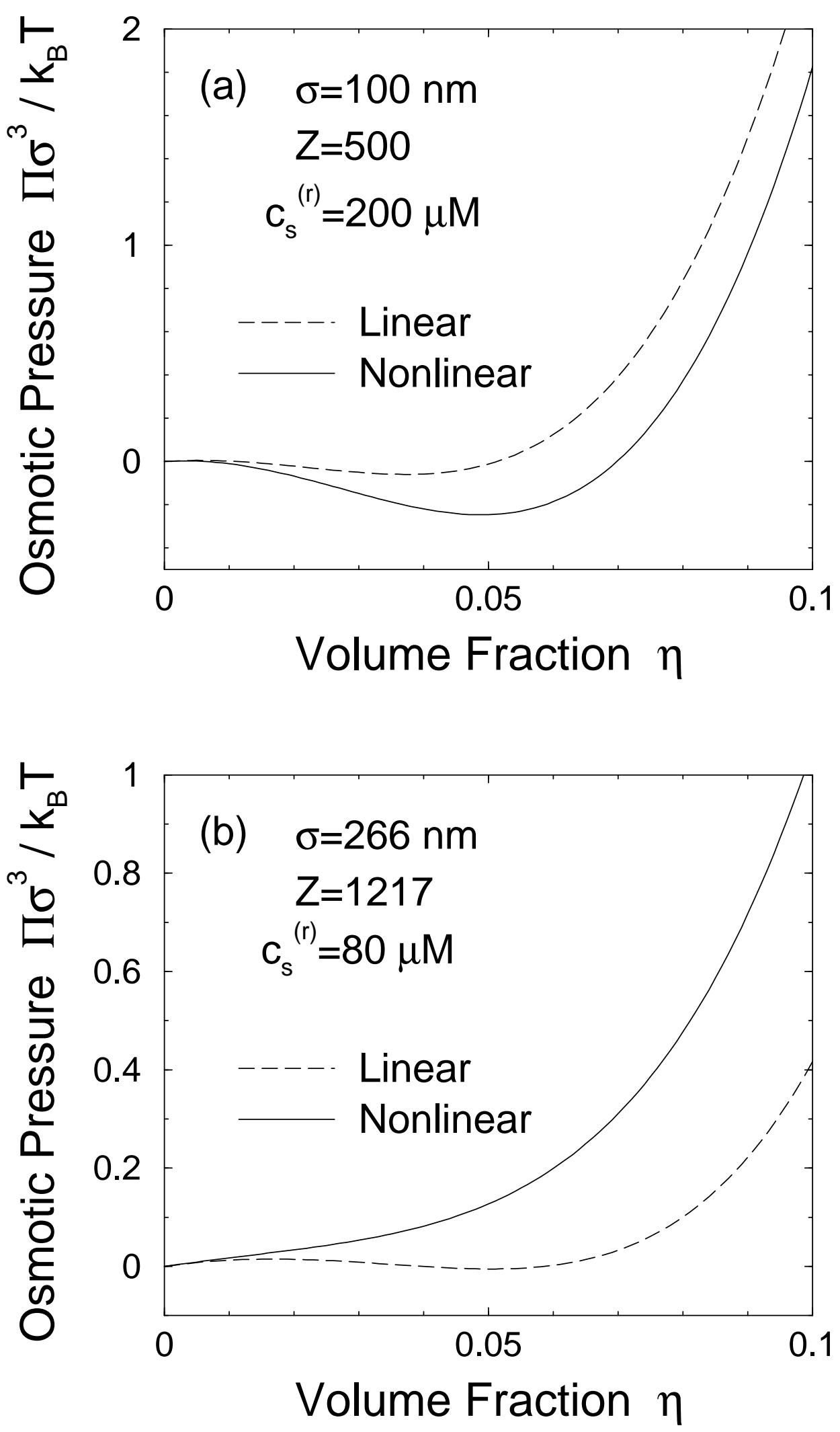


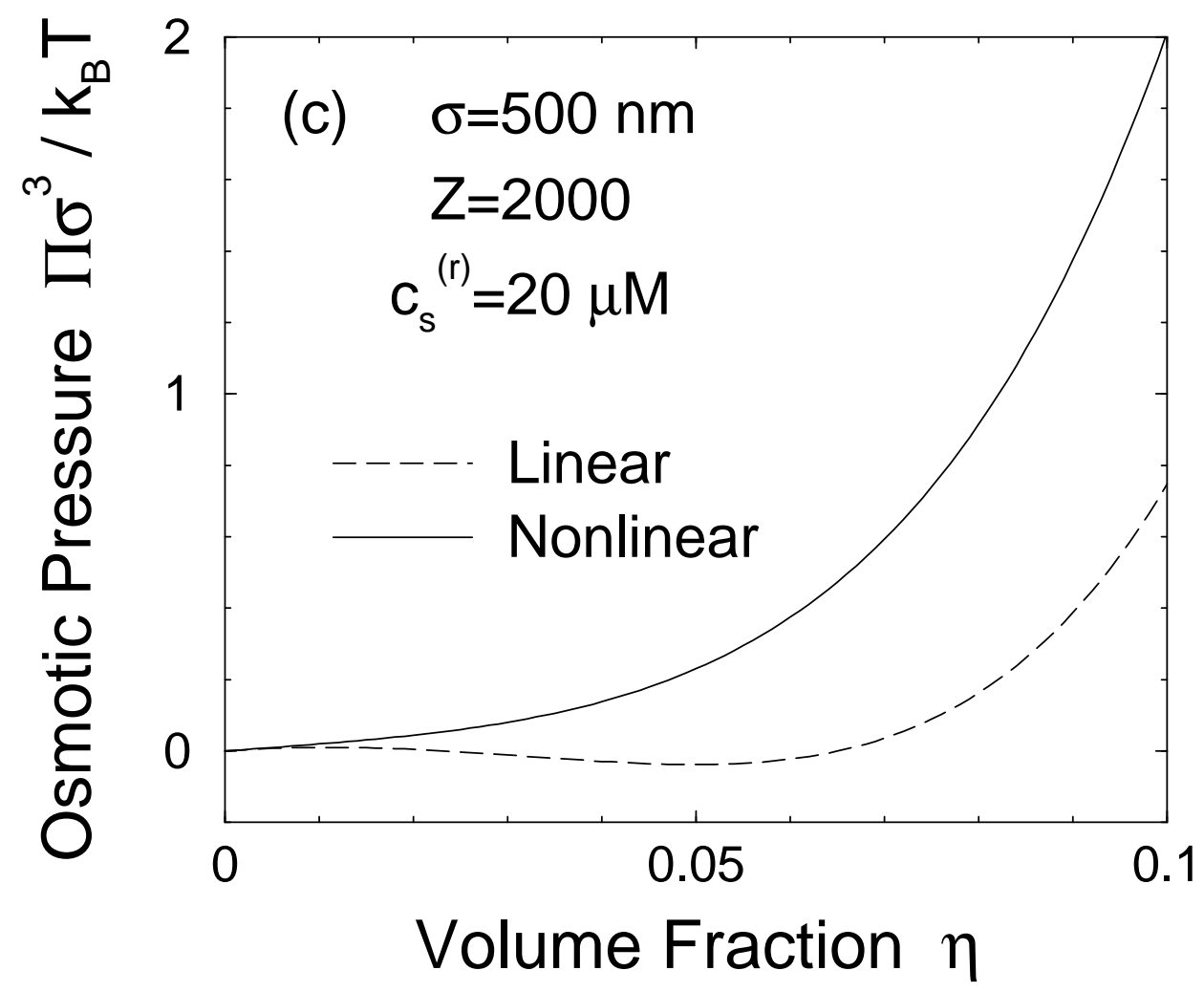

FIG. 4: Osmotic pressure $\Pi$ (in reduced units) vs. colloid volume fraction $\eta$ for same combinations of macroion diameter $\sigma$ and valence $Z$ as in Fig. 2 and fixed reservoir salt concentration $c_{s}^{(r)}$ : (a) $\sigma=100 \mathrm{~nm}, Z=500$, $c_{s}^{(r)}=200 \mu \mathrm{M}$; (b) $\sigma=266 \mathrm{~nm}, Z=1217, c_{s}^{(r)}=80 \mu \mathrm{M}$; (c) $\sigma=500 \mathrm{~nm}, Z=2000, c_{s}^{(r)}=20 \mu \mathrm{M}$. Solid (dashed) curves are predictions of nonlinear (linear) response theory. 

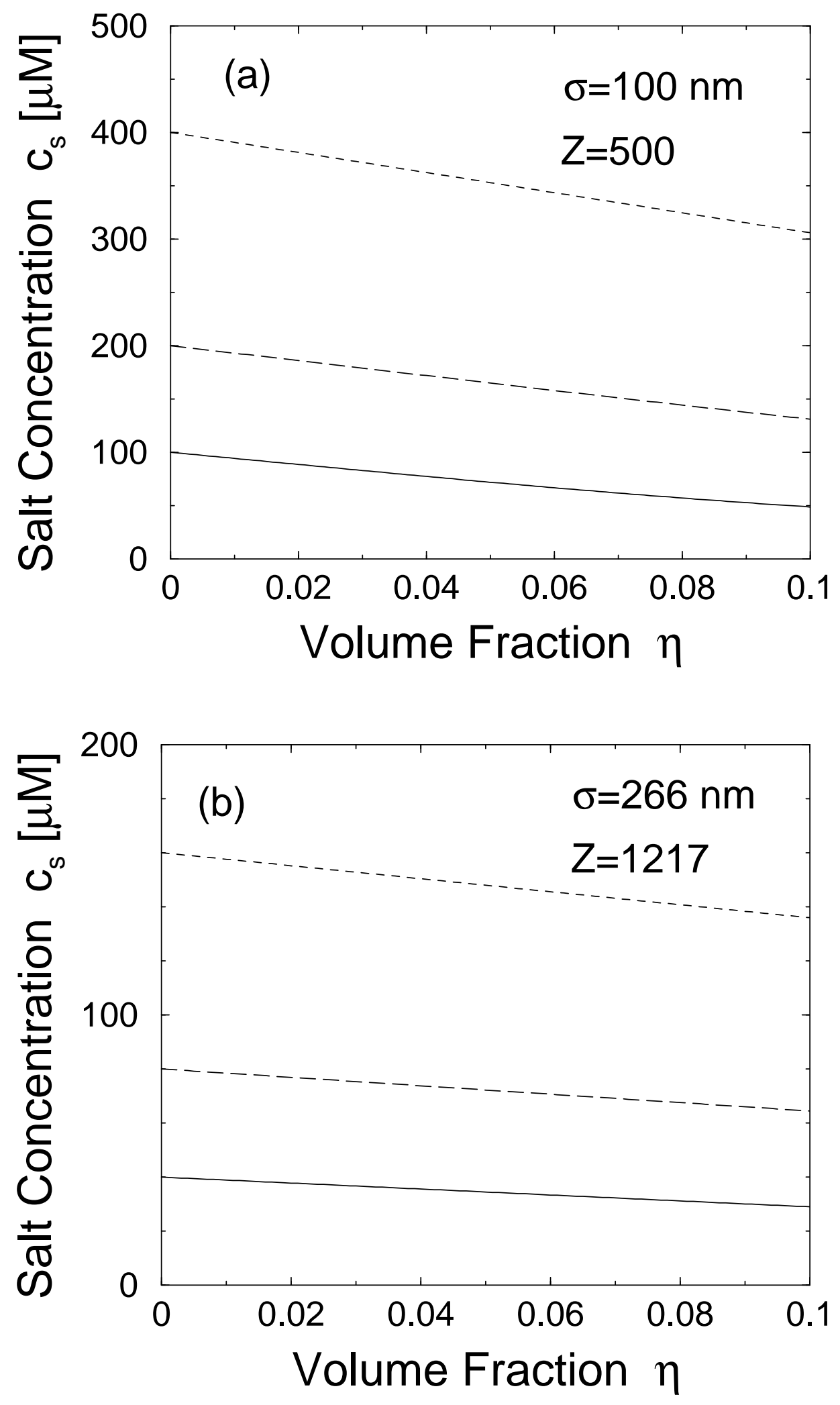


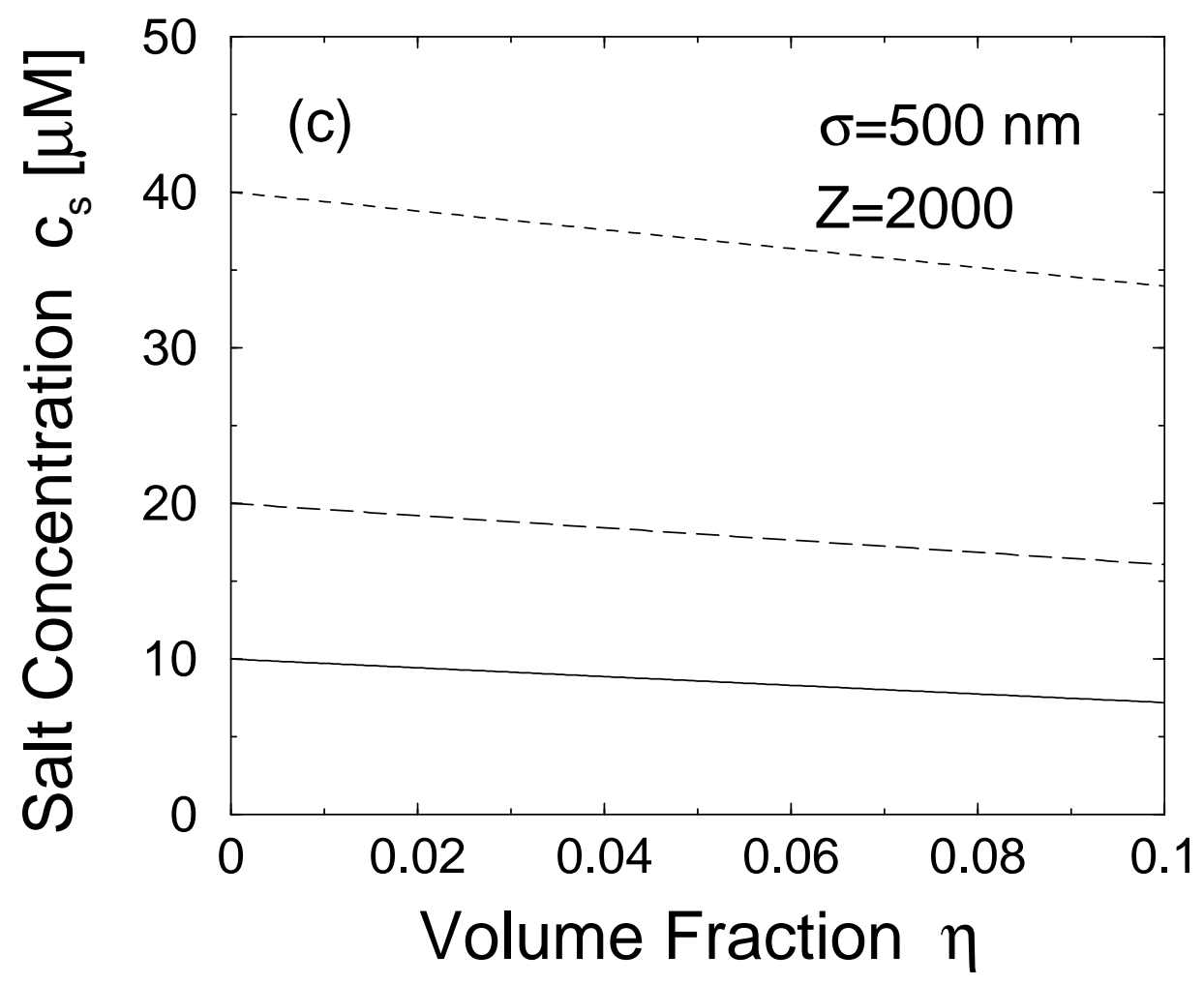

FIG. 5: Linear-screening predictions for system salt concentration $c_{s}[\mu \mathrm{mol} / \mathrm{liter}]$ vs. colloid volume fraction $\eta$ for same system parameters as in Fig. 2 at various fixed salt chemical potentials. Respective reservoir salt concentrations are given by intersections of curves with $\eta=0$ axis. 

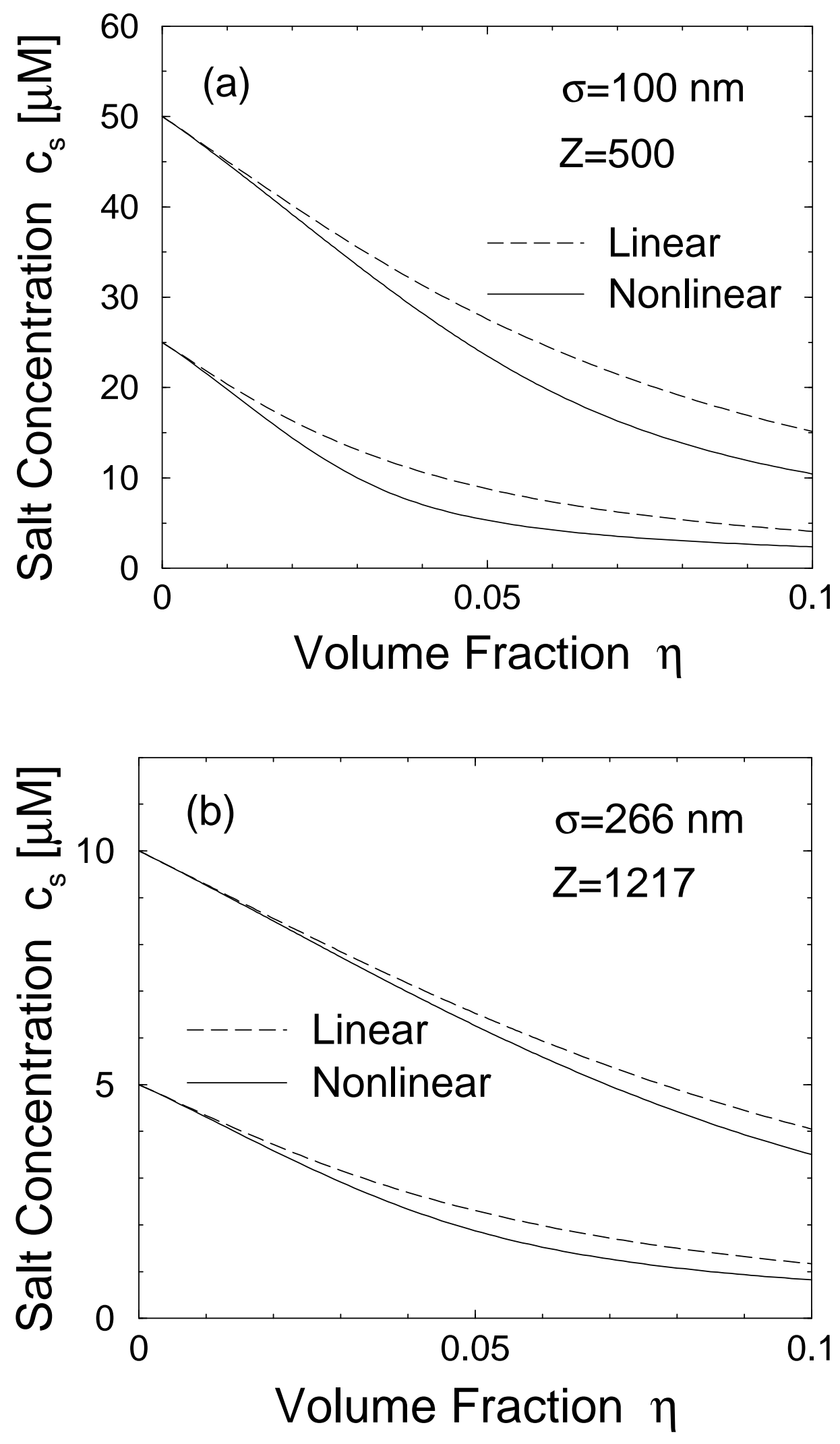


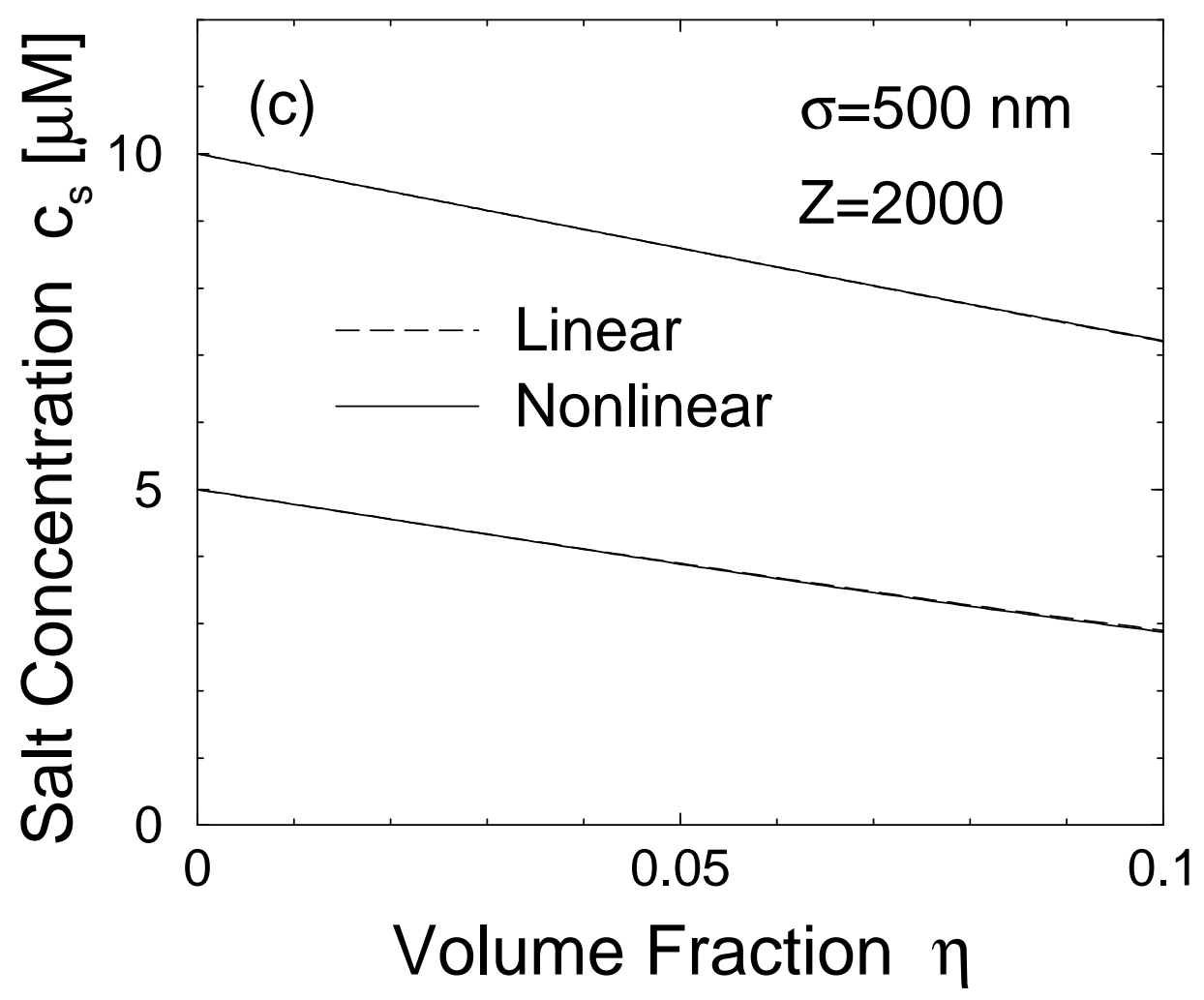

FIG. 6: Linear- and nonlinear-screening predictions for system salt concentration $c_{s}[\mu \mathrm{mol} / \mathrm{liter}]$ vs. colloid volume fraction $\eta$ for same system parameters as in Fig. 2 and at two fixed salt chemical potentials. Respective reservoir salt concentrations are given by intersections of curves with $\eta=0$ axis. Solid (dashed) curves are predictions of linear (nonlinear) response theory. 

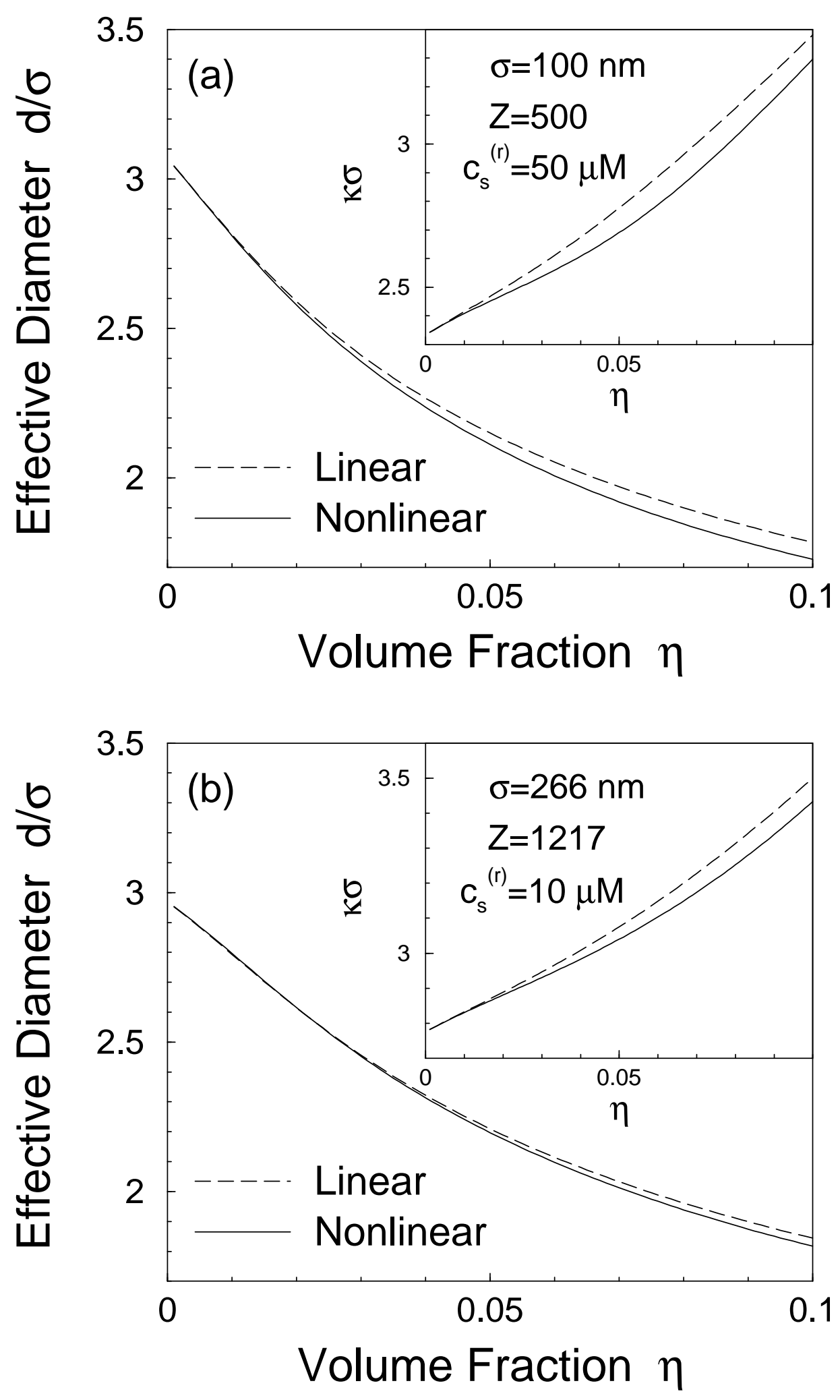


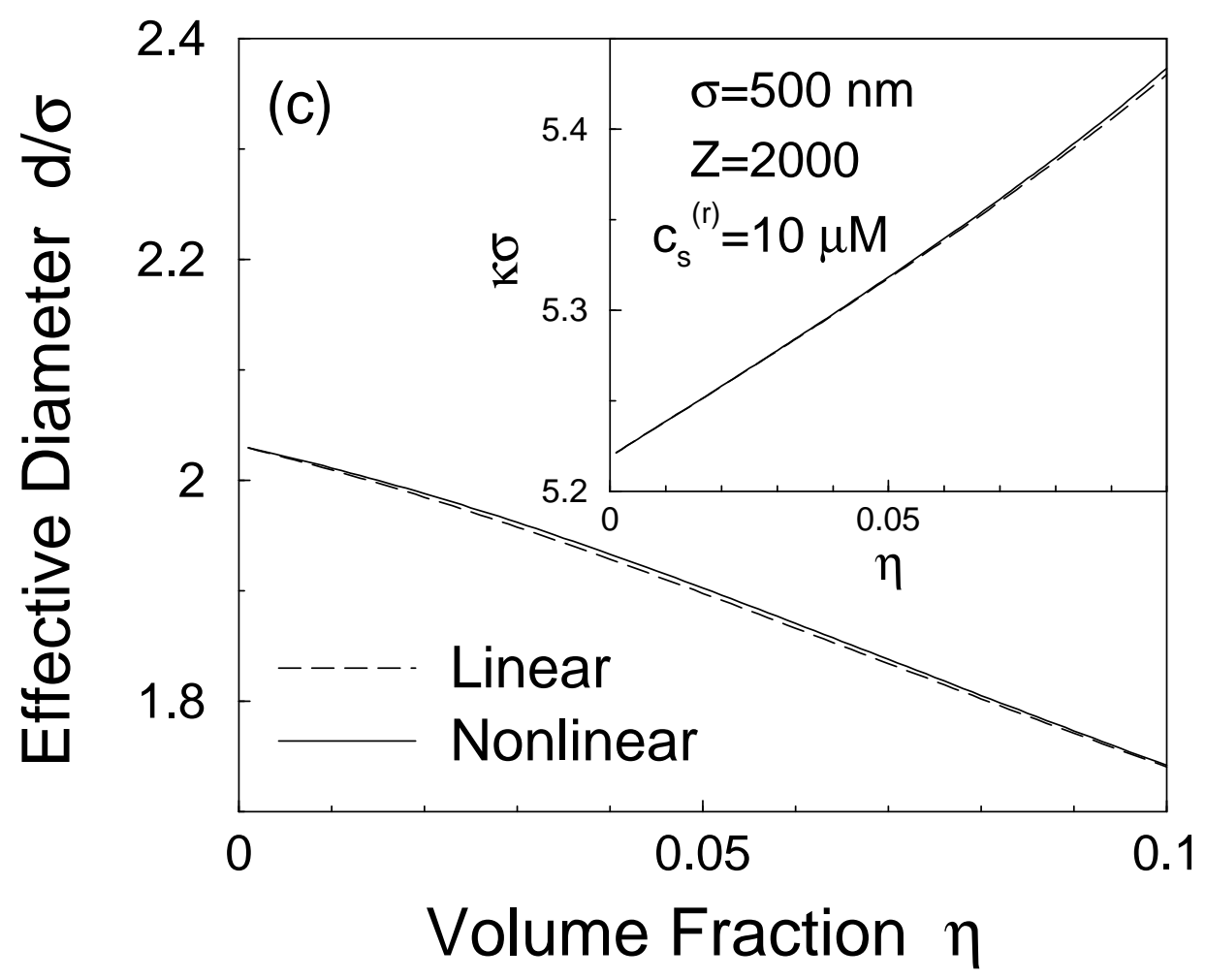

FIG. 7: Effective hard-sphere diameter $d$ (units of macroion diameter $\sigma$ ) and Debye screening constant $\kappa$ (inset) vs. colloid volume fraction $\eta$, at fixed reservoir salt concentration $c_{s}^{(r)}$, for (a) $\sigma=100 \mathrm{~nm}, Z=500, c_{s}^{(r)}=50 \mu \mathrm{M}$; (b) $\sigma=266 \mathrm{~nm}, Z=1217, c_{s}^{(r)}=10 \mu \mathrm{M}$; (c) $\sigma=500 \mathrm{~nm}, Z=2000, c_{s}^{(r)}=10 \mu \mathrm{M}$. Solid (dashed) curves are predictions of nonlinear (linear) response theory. 

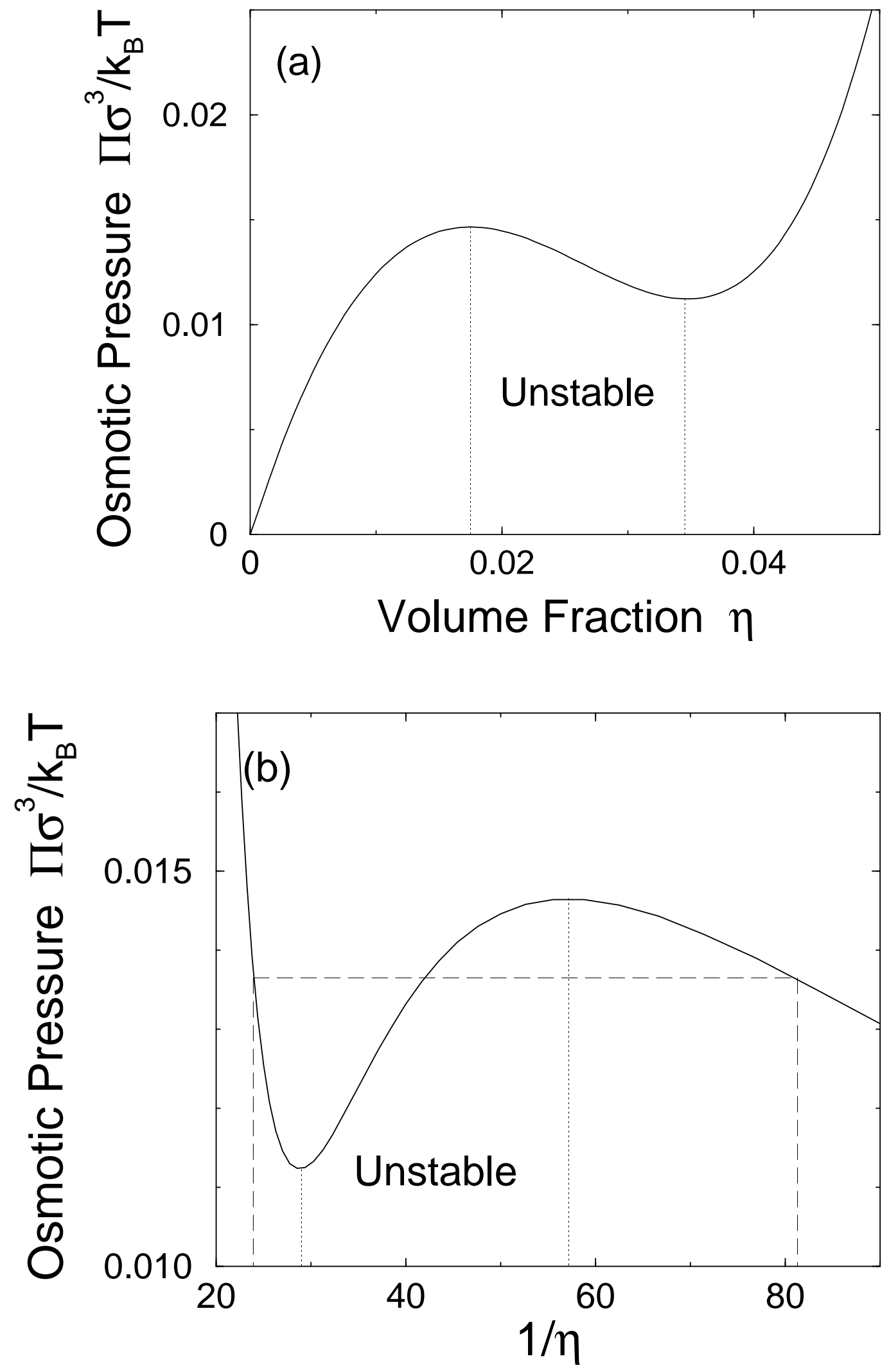


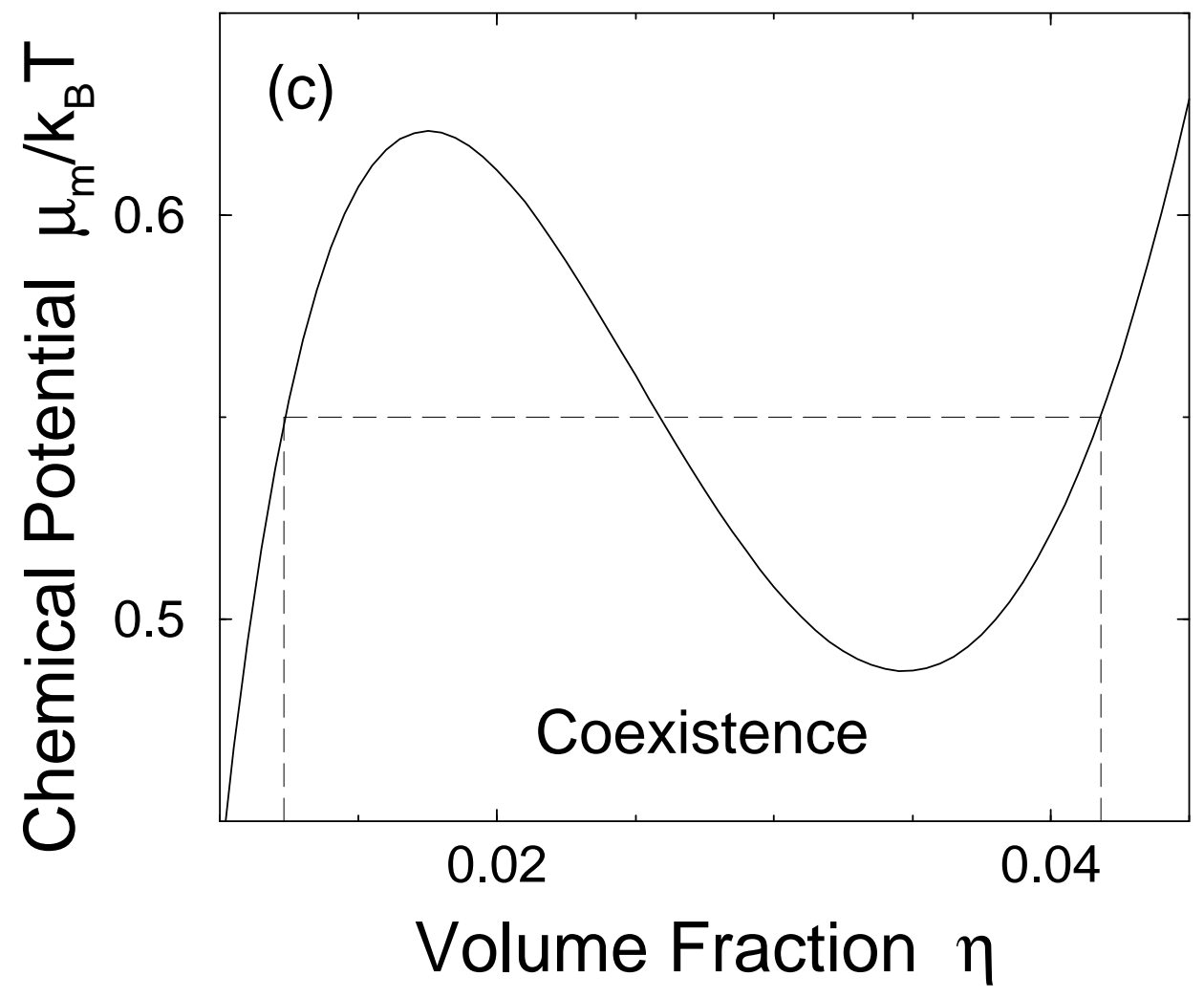

FIG. 8: Linear-screening prediction for (a) osmotic pressure $\Pi$ vs. colloid volume fraction $\eta$, (b) $\Pi$ vs. $1 / \eta$, and (c) colloid chemical potential $\mu_{m}$ (shifted by arbitrary constant) vs. $\eta$ for macroion diameter $\sigma=100 \mathrm{~nm}$, valence $Z=500$, and reservoir salt concentration $c_{s}^{(r)}=350 \mu \mathrm{M}$. In panels (a) and (b), dotted vertical lines at maximum and minimum of $\Pi$ indicate spinodal densities at boundaries of unstable region. In panels (b) and (c), dashed vertical lines indicate coexisting densities on the fluid binodal, illustrating the Maxwell equal-area construction. 

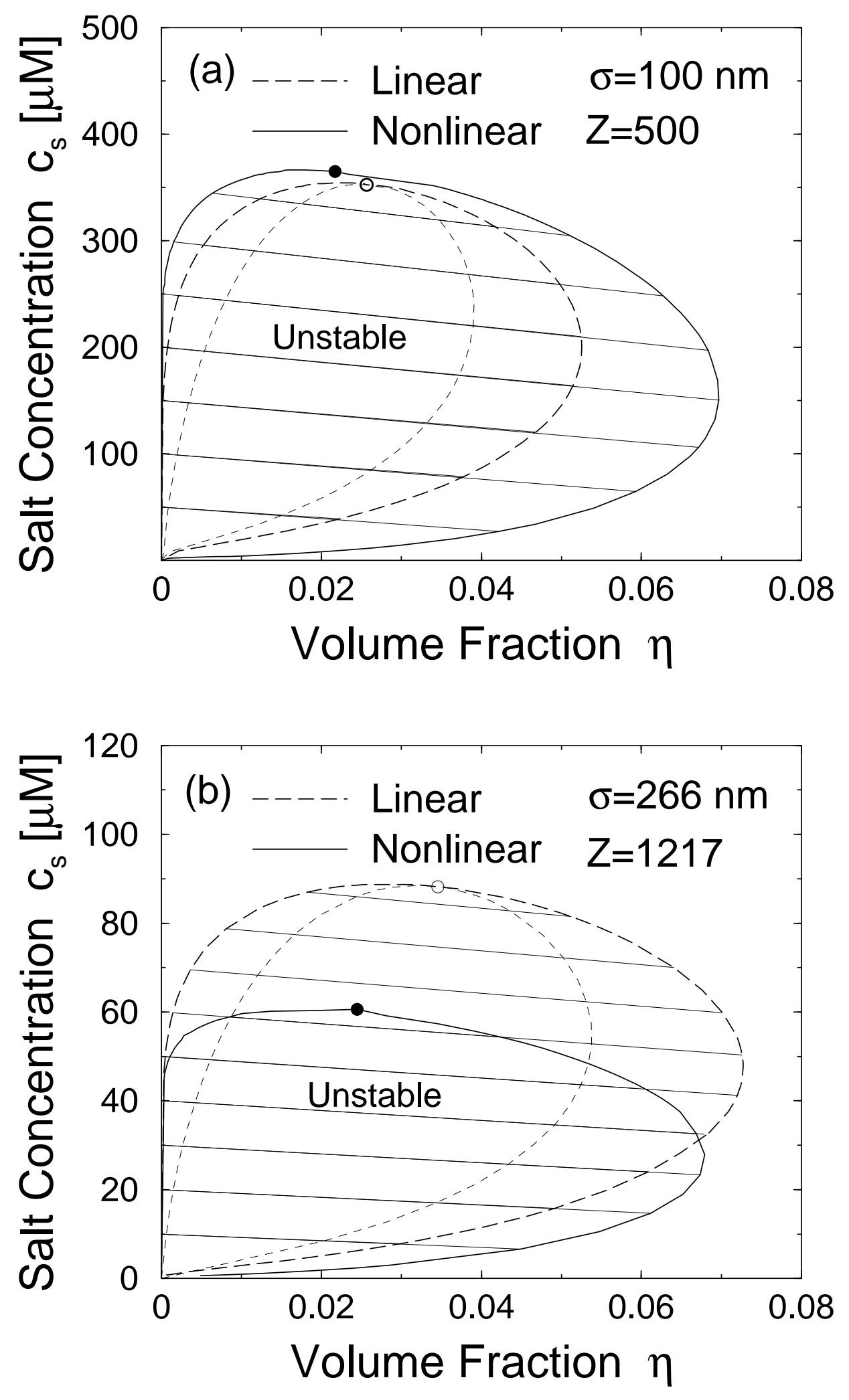


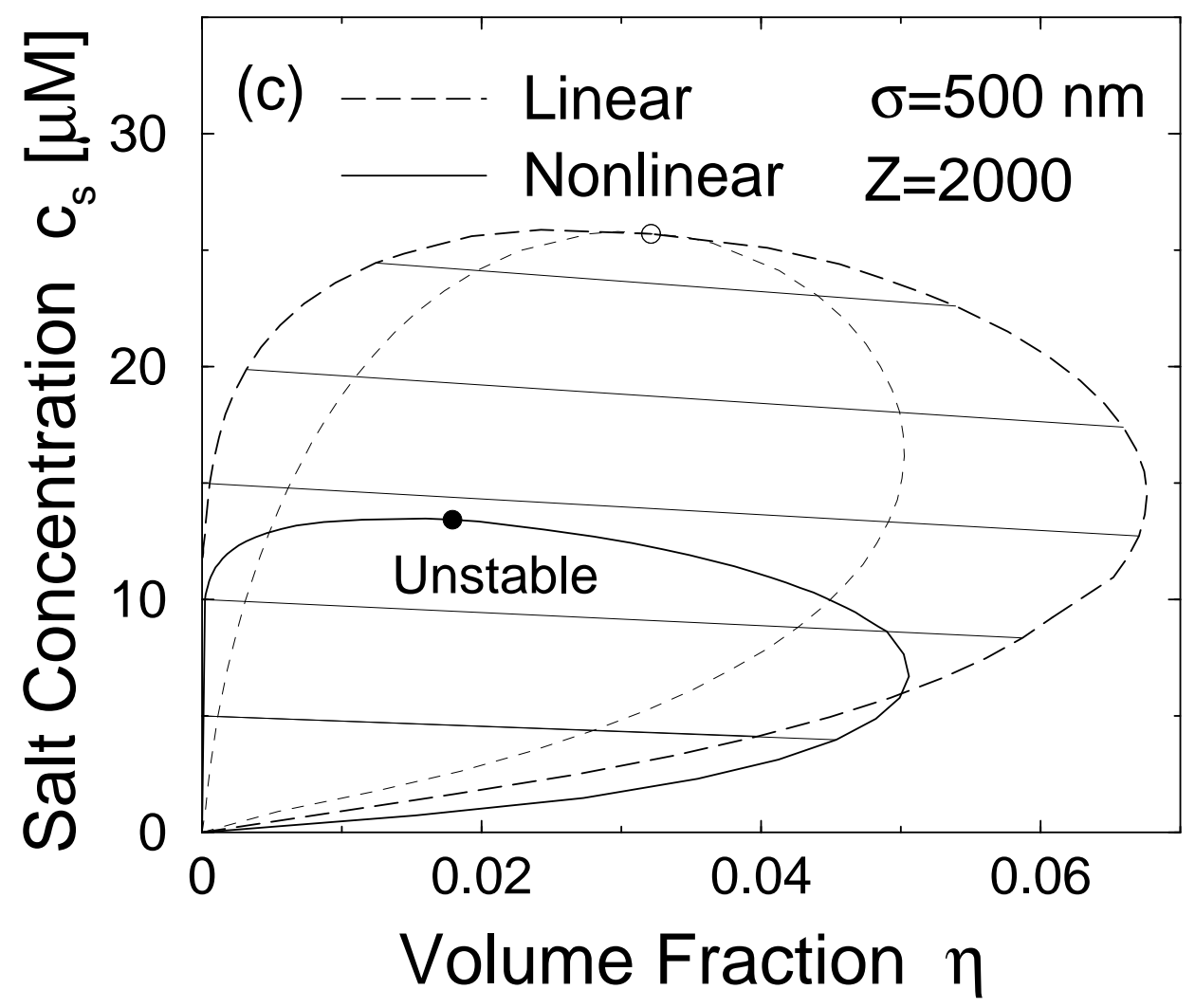

FIG. 9: Fluid phase diagrams for aqueous suspensions of charged colloids at room temperature $\left(\lambda_{B}=0.72 \mathrm{~nm}\right)$ with monovalent microions and various macroion diameters and effective valences: (a) $\sigma=100 \mathrm{~nm}, Z=500$; (b) $\sigma=266 \mathrm{~nm}, Z=1217$; (c) $\sigma=500 \mathrm{~nm}, Z=2000$. Solid (long-dashed) curves represent predictions for binodals from nonlinear (linear) response theory. Short-dashed curves represent predictions for spinodals (linear response only). Circular symbols denote critical points. Tie lines join corresponding points on liquid and vapor branches of binodals. 